1 Close Cassini Flybys of Saturn's Ring Moons Pan, Daphnis, Atlas, Pandora, and

2 Epimetheus

3 B. J. Buratti ${ }^{1}$, P. C. Thomas ${ }^{2}$, E. Roussos ${ }^{3}$, C. Howett ${ }^{4}$, M. Seiß ${ }^{5}$, A. R. Hendrix ${ }^{6}$, P. Helfenstein ${ }^{2}$, R. H. 4 Brown $^{7}$, R. N. Clark ${ }^{6}$, T. Denk ${ }^{8}$, G. Filacchione ${ }^{9}$, H. Hoffmann ${ }^{5}$, G. H. Jones ${ }^{10}$, N. Khawaja ${ }^{11}$, P. Kollmann ${ }^{12}$, $5 \quad$ N. Krupp ${ }^{3}$, J. Lunine ${ }^{2}$, T. W. Momary ${ }^{1}$, C. Paranicas ${ }^{12}$, F. Postberg ${ }^{11}$, M. Sachse ${ }^{5}$, F. Spahn ${ }^{5}$, J. Spencer ${ }^{4}$, R. 6 Srama $^{13}$, T. Albin ${ }^{13}$, K. H. Baines ${ }^{1}$, M. Ciarniello ${ }^{9}$, T. Economou ${ }^{14}$, H.-W. Hsu ${ }^{15}$, S. Kempf ${ }^{15}$, S. M. Krimigis ${ }^{12}$, 7 D. Mitchell ${ }^{12}$, G. Moragas-Klostermeyer ${ }^{13}$, P. D. Nicholson ${ }^{2}$, C. C. Porco ${ }^{16}$, H. Rosenberg ${ }^{8}$, J. Simolka ${ }^{13}$, L. A. 8 Soderblom $^{17}$

91 Jet Propulsion Laboratory, California Institute of Technology, Pasadena, CA 91109, USA

$10{ }^{2}$ Cornell Center for Astrophysics and Planetary Science, , Cornell University, Ithaca, NY 14853, USA

$11{ }^{3}$ Max Planck Institute for Solar System Research, 37077 Göttingen, Germany

12 4Southwest Research Institute, Boulder, CO 80302, USA

${ }^{5}$ Department of Physics and Astronomy, University of Potsdam, 14476 Potsdam, Germany

${ }^{6}$ Planetary Sciences Institute, Tucson, AZ 85719, USA

${ }^{7}$ Lunar and Planetary Lab, University of Arizona, Tucson, AZ 85721, USA

${ }^{8}$ Freie Universität Berlin, 12249 Berlin, Germany

${ }^{9}$ Istituto di Astrofisica e Planetologia, Via Fosso del Cavaliere 100, Rome, Italy

${ }^{10}$ University College London, London, UK

${ }^{11}$ Institut für Geowissenschaften, Universität Heidelberg, 69120 Heidelberg, Germany Germany and Institut für Geologische Wissenschaften, Freie Universität, 12249 Berlin, Germany

${ }^{12}$ Applied Physics Laboratory, Johns Hopkins University, Laurel, MD 20723, USA

${ }^{13}$ University of Stuttgart, Pfaffenwaldring 29, 70569 Stuttgart, Germany

${ }^{14}$ Enrico Fermi Institute, University of Chicago, Chicago, IL, 60637, USA

15 Physics Department, University of Colorado, Boulder, CO 80303, USA

${ }^{16}$ Space Sciences Institute, Boulder CO 80301, USA

${ }^{17}$ United States Geological Survey, Flagstaff, AZ 86001, USA

Submitted to Science Feb. 6 for the Cassini End-of Mission special issue Resubmitted May 24, 2018

Resubmitted February 14, 2019 
Abstract

40

41

Saturn's main ring system is associated with a unique set of small moons that are either embedded within it, or interact with the rings to alter their shape and composition. Six close flybys of Pan, Daphnis, Atlas, Pandora, and Epimetheus were performed between December 2016 and April 2017 during the Ring-grazing Orbits of the Cassini mission. Data on the moons' morphology, structure, particle environment, and composition were returned, as well as images in the ultraviolet and thermal infrared. The optical properties of the moons' surfaces are determined by two competing processes: contamination by a red material formed in Saturn's main ring system, and by accretion of bright icy particles or water vapor from volcanic plumes originating on the planet's moon Enceladus.

Saturn possesses a family of small inner irregular moons that orbit close to its rings. Two moons orbit in gaps within Saturn's main ring system: Daphnis, which dwells in the A-ring's Keeler gap (1), and Pan, which is found in the Encke gap, also in the A-ring (2). Three others, called shepherd moons, orbit at the edges of the A-ring (Atlas) or the F-ring (Pandora and Prometheus) (supplementary materials, Fig. S2) Co-orbital moons Janus and Epimetheus share horse-shoe orbits outside the F-ring and swap their positions every four years (Fig. S2). Saturn's rings are almost certainly tied to the origin and continued existence of these moons (1). It remains unclear whether the rings formed from the break-up of an inner moon, or if the present ring moons formed from the consolidation of existing ring material, either primordial or impact-created. The alteration processes acting on these moons and the rings, past and present, are also unknown. Prior to 
Saturn's exploration by spacecraft, the main rings were thought to be unconsolidated primordial debris, unable to form a moon because of tidal forces $(3,4)$. Evidence from the two Voyager spacecraft suggested the rings and inner moons were both debris from the breakup of a single parent body, or perhaps several parent bodies, with the moons being the largest fragments from the collision (3). Measurement of the rings' and moons' bulk densities using Cassini data (4), along with dynamical studies, and the existence of ridges around the equators of Atlas and Pan $(4,5)$, suggested a more complicated, multi-stage formation. The ring moons - from Pan out to Pandora, but possibly also Janus and Epimetheus - likely formed from the very early accretion of low density debris around a denser seed, presumably a collisional shard from the breakup of a preexisting moon (4). In the cases of Atlas and Pan, this was followed by a second stage of accretion of material onto the equator, after the rings had settled into their present very thin disk (5-6). In this scenario, the surfaces of these moons should be similar in composition to the rings.

Analysis of the optical properties of the moons including color, albedo, and spectral properties in the visible and infrared between 0.35 and $5.2 \mu \mathrm{m}$ has shown that they resemble the ring systems in which they are embedded or abut (7-10). An unidentified low-albedo reddish material that could be organic molecules, silicates, or iron particles (8-11) appears to be abundant in the rings and has also tinged the moons (7-11), further supporting a common origin and implying continuing accretion of particles onto the moons' surfaces. The interactions of the ring system with the inner moons may form two distinct zones: an inner region in the vicinity of the main ring system that is dominated by the red chromophore, and an outer region that is dominated by fresh, high albedo icy particles from the E-ring. Complicating the picture, however, is the possible influence of interactions with magnetospheric particles, which have been shown to alter the color and albedo 
of the main moon system of Saturn $(12,13)$. It is unclear whether any volatiles other than water ice exist on the ring moons. The presence of molecules with higher volatility than water ice would indicate material originating in a colder region outside the Saturnian system; for example, the discovery of $\mathrm{CO}_{2}$ ice on the irregular outer moon Phoebe suggested that it originated in the Kuiper Belt (14).

The last phase of Cassini's mission began on November 30, 2016 and ended on September 15, 2017, with two distinct periods: the Ring-grazing (or F-ring) Orbits, in which 20 close passes to the F-ring were performed, and the Proximal Orbits (or Grand Finale), which executed 23 dives between the planet and the main ring system. During the Ring-grazing Orbits Cassini performed its closest flybys of Pan, Daphnis, Atlas, Pandora, and Epimetheus (Table 1). A second flyby of Epimetheus was performed at a slightly greater distance. Data were obtained using several instruments on Cassini: The Imaging Science Subsystem (ISS; 15); The Visual Infrared Mapping Spectrometer, taking medium resolution spectra between 0.35 and $5.1 \mu \mathrm{m}$ (VIMS; 16); The Cassini Infrared Spectrometer (CIRS; 17); The Ultraviolet Imaging Spectrometer (UVIS; 18); the Cosmic Dust Analyzer (CDA; 19) and the Magnetosphere Imaging Instrument (MIMI; 20). The dust and plasma environment in the vicinity of the small inner moons was observed by the particles instruments during the subsequent Proximal Orbits.

[Table 1 here]

\section{Geology and morphology}

Previous images of the ring moons showed distinctive equatorial ridges on Pan and Atlas $(4,5)$ which were interpreted as likely formed by accretion of ring particles, whilst those of Daphnis were ambiguous. The small satellites are all in synchronous rotation, tidally locked to the planet (6). Prometheus and Pandora's orbits straddle the F-ring, and although they exhibit different 
surface morphology, their densities are nearly identical (Supplementary materials, Table S1). The small $(<5 \mathrm{~km}$ mean radius) satellites Aegaeon, Methone, and Pallene that orbit in diffuse rings or ring $\operatorname{arcs}(21,22)$ have smooth ellipsoidal shapes indicative of hydrostatic equilibrium (6). The coorbital satellites, Epimetheus and Janus, by far the largest of the inner small moons, have nearly identical mean densities (Table S1), which are also the highest among the inner small moons. Grooves had been observed on Epimetheus (23), and there were suggestions of discrete craterfilling sediments on both Janus and Epimetheus (6). Epimetheus experiences a $\sim 7^{\circ}$ forced wobble (libration) around a purely synchronous rotation (24). Table S1 summarizes the shapes, volumes, and calculated mean densities of the small satellites of Saturn based on the images taken during the flybys $(25,26)$. Epimetheus and Janus have densities substantially above $500 \mathrm{~kg} \mathrm{~m}^{-3}$; the lowest density (and highest uncertainty) is that of Daphnis, at $274 \pm 142 \mathrm{~kg} \mathrm{~m}^{-3}$. Surface accelerations vary substantially across each object due to their irregular shapes and tidal accelerations (Table S1).

\section{Main Ring moons and ridges}

The flyby images in Fig. 1 show that the equatorial ridges on Pan and Atlas are morphologically distinct from the more rounded central component of each moon. The ridges are different sizes on each moon: the fractional volumes of the ridges are Pan $\sim 10 \%$; Daphnis $\sim 1 \%$, and Atlas $\sim 25 \%$. Atlas's ridge appears smooth in the highest resolution image (76 m/pixel), with some elongate brighter albedo markings. The ridge contacts the central component that has distinct ridge and groove topography (Fig. 1C); it has a previously known slight polygonal equatorial profile (6). Pan's ridge has a distinct boundary with the central component, a somewhat polygonal equatorial shape, some grooves, small ridges, and even several small impact craters. The profile of Pan's ridge varies considerably with longitude. Fig. 2 shows Pan's northern hemisphere, with calculated relative gravitational topography and surface slopes using existing techniques $(4,6)$ 
129 (supplementary material, data file of Pan's gravity). Unlike some equatorial ridges on small 130 asteroids $(27,28)$, Pan's ridge was not formed by material sliding toward lower gravitational 131 potential areas generated by rotation and tides, because the slope directions are not latitudinally 132 directed. The distinct boundary between ridge and central competent core, the differing surface 133 morphology on each, and the large differences in relative heights along the ridge require the ridge 134 formation to be unrelated to surface, gravity-driven processes. These observations are consistent 135 with formation of the ridge by the accretion of particles, with a distribution dictated by the relative 136 orbital and rotational dynamics of the moon and ring particles (5). 

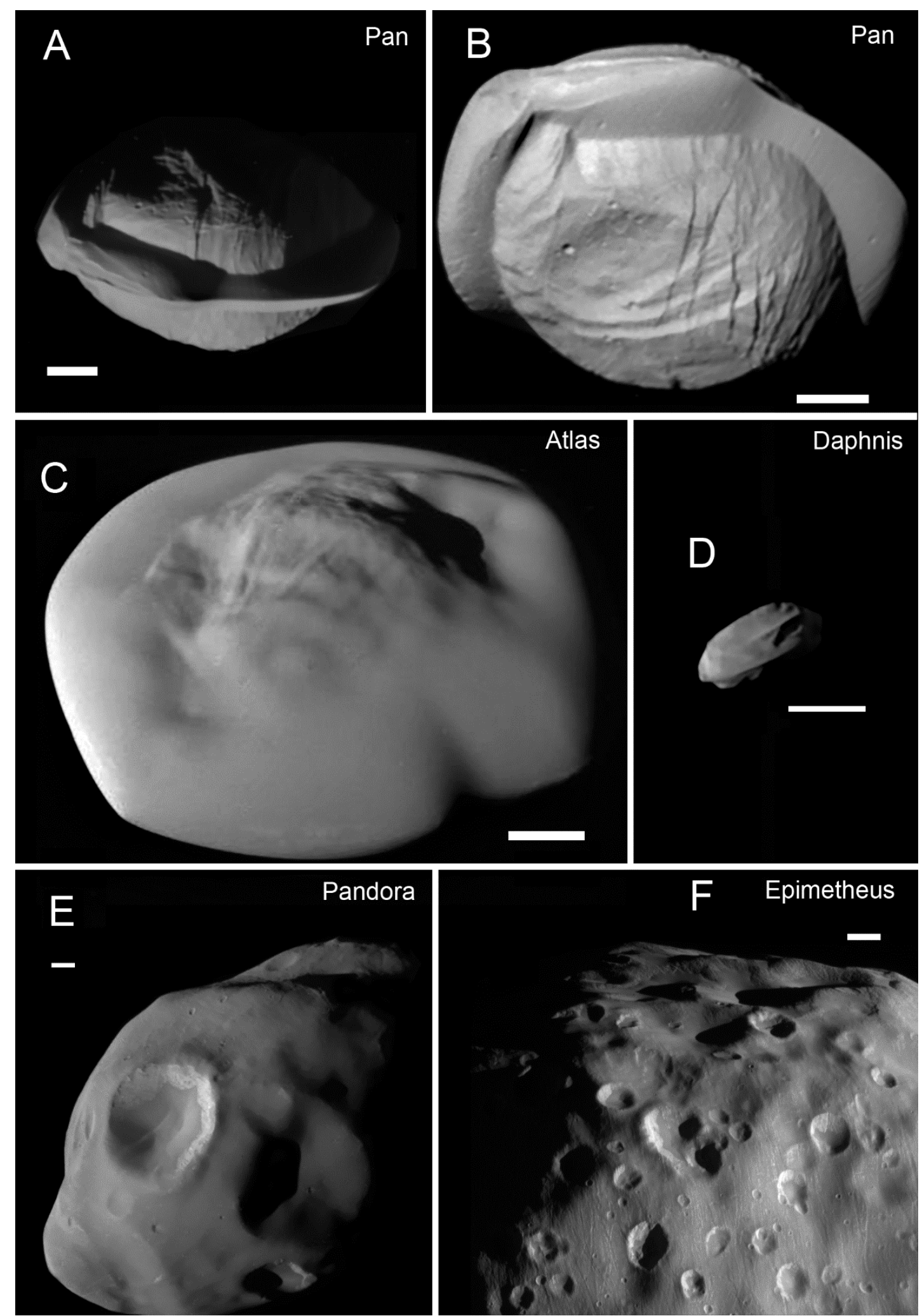
Fig 1. Greyscale images of the ring moons obtained with ISS during the Cassini flybys. (A) Pan, image number N1867606181, Clear/Clear filters, from $26^{\circ} \mathrm{S}$, at a scale of 182 meters per pixel (m/pix). (B) Pan, N186704669, Clear/Clear filters, from $39^{\circ} \mathrm{N} ; 147 \mathrm{~m} / \mathrm{pix}$. (C) Atlas, N1870699087, Clear/IR3 filters, from $40^{\circ} \mathrm{N}$; anti-Saturn point at lower left; $108 \mathrm{~m} /$ pix. (D) Daphnis, N1863267232, Clear/Green filters, from $14^{\circ} \mathrm{N}$; anti-Saturn point to left; $170 \mathrm{~m} / \mathrm{pix}$. (E) Pandora N1860790629, Clear/Green filters. The sub-spacecraft point is $35^{\circ} \mathrm{N}, 98^{\circ} \mathrm{W}$; Pandora's north pole is close to two small craters above the large, bright-walled crater; $240 \mathrm{~m} / \mathrm{pix}$. (F) Epimetheus N1866365809, Clear/UV3 filters; Grooves and craters dominate the surface; 99 m/pix. All scale bars are $5 \mathrm{~km}$. Images were chosen for scale and viewing geometry; different filters have little effect on visibility of morphologic detail.
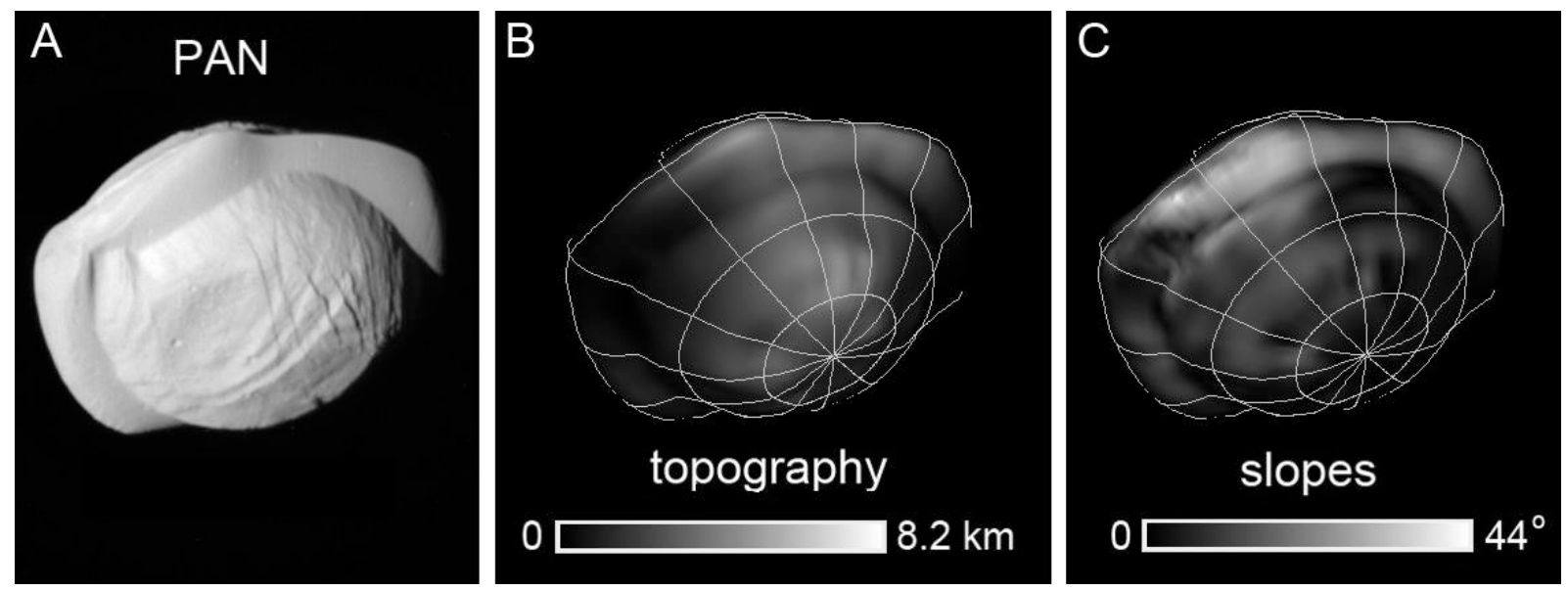

Fig. 2. Relative topography and slopes on Pan. A) Greyscale image N1867604669 from $39^{\circ} \mathrm{N}$, $217^{\circ} \mathrm{W}$ (rotated from view in Fig.1). B) Topography is the dynamic topography which is the relative potential energy at the surface (due to mass, rotation, and tides) divided by an average surface acceleration $(4,6)$. A homogeneous interior density is assumed. C) Slopes are the angles between the surface normals and the (negative) net acceleration vectors. 
The calculated mean densities of Pan, Atlas and Daphnis result in calculated surface accelerations near zero at the sub- and anti-Saturn points, suggesting those points cannot accrete additional material. The rest of the surfaces have inward-directed net accelerations. The surfaces of these three moons may be crudely divided into three units on the basis of morphology, geography, and surface texture visible at the available resolutions (Fig. 3). The equatorial ridges generally are the smoothest terrain on each moon.

The central components have more impact craters than do the ridges on Pan and Atlas, which display a few sub-kilometer impact craters. Pan and Atlas's central components show lineated topography indicative of structures such as faults or fractures.. Pan has two distinct global sets of quasi-parallel faults. The first is roughly concentric to the long axis and exhibits conspicuous scarps and terracing, likely formed by equatorward displacements. Axial symmetry of this system suggests that tidal forces were involved in its development. The second system is oriented obliquely to the first, and is visible in both north and south hemispheres (Figs.1A, 3C). In contrast, Atlas's central component core exhibits patterns of elongated ridge and groove topography that do not have fault scarp morphologies and appears to be covered by at least tens of meters of loose material (regolith).

Pan's equatorial ridge is thickest north-south at longitudes of approximately $220^{\circ}, 310^{\circ}, 135^{\circ}$, and $50^{\circ} \mathrm{W}$, yet its radial extent peaks at longitudes of about $5^{\circ}, 55^{\circ}, 100^{\circ}, 180^{\circ}, 235^{\circ}$, and $310^{\circ}$. The ridge supports grooves and small craters: their presence suggests some cohesion in this low-gravity environment (less than $2 \mathrm{mms}^{-2}$ ). Atlas's equatorial profile is also somewhat polygonal, but not as pronounced as Pan's. 

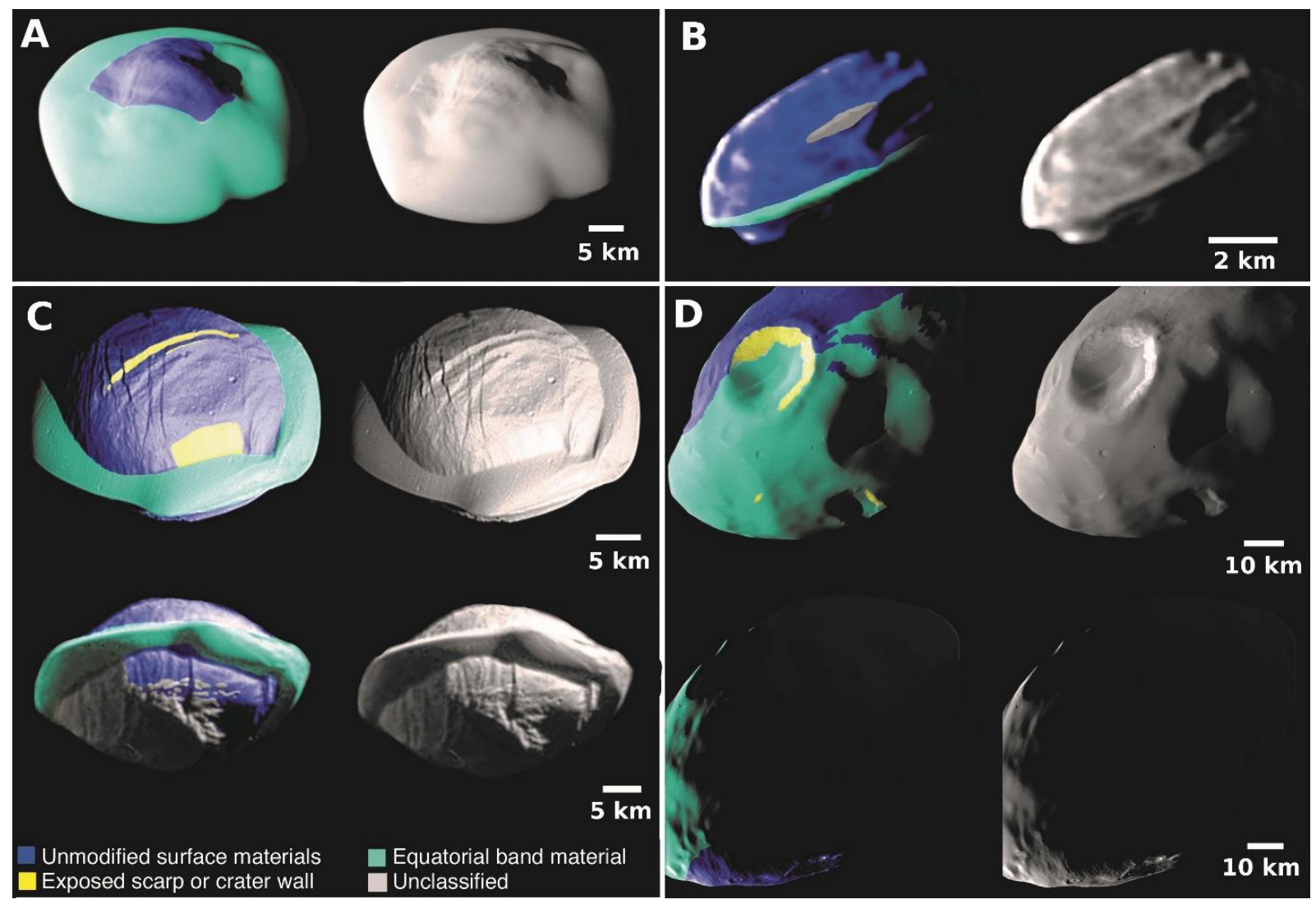

Fig. 3. Distribution of geological units on Pan, Atlas, Daphnis and Pandora. In each panel the three main units are highlighted in color, with the uninterpreted greyscale image alongside for comparison. Cratered surfaces (blue) have numerous craters, relatively crisp surface relief, and regolith typical of other small bodies in the Saturnian system. Smooth terrains (cyan) are distinctly smooth compared to typical small body cratered surfaces; some is material collected in crater floors. Exposed substrates (yellow) are relatively bright with lineations, more typical of rigid materials than of loose regolith. Unclassified areas (grey) are those for which insufficient data are available to resolve ambiguities between terrain types. (A) Atlas, scale bar $5 \mathrm{~km}$, resolution 94 m/pix. (B) Daphnis, scale bar 2km; 167 m/pix. (C) Pan, scale bars 5 km; 144 m/pix (top) and 279 m/pix (bottom). (D) Pandora (top scalebar, 10km, bottom, $20 \mathrm{~km}$ ); $137 \mathrm{~m} / \mathrm{p}$ (top), $200 \mathrm{~m} / \mathrm{p}$ (bottom). 
195 The classification of some material units on Pan's southern hemisphere is ambiguous, in part 196 because these are not directly illuminated by the Sun, only by light reflected off Saturn. These 197 unclassified units in Fig. 3C include knobby streaks of hummocked material orientated 198 approximately parallel to the equator and hummocky deposits outlining a curvilinear depression 199 on the Saturn-facing side.

The spatial resolution of the Daphnis imagery is $170 \mathrm{~m} /$ pixel, poorer than that of Pan $(147 \mathrm{~m} / \mathrm{pixel})$ and Atlas (76 m/pixel). Daphnis is only about a quarter the dimensions of the other ring moons. As a result, it is not clear whether its near-equatorial ridge is smoother or otherwise different from the rest of the surface. The equatorial ridge extends at least from $75^{\circ} \mathrm{W}$ to $185^{\circ} \mathrm{W}$. An additional ridge at $22^{\circ} \mathrm{N}$ runs from $\sim 60^{\circ} \mathrm{W}$ to $120^{\circ} \mathrm{W}$. Both ridges are $300-400 \mathrm{~m}$ north-south, and perhaps radially $300 \mathrm{~m}$ in extent. The core has an elongated $(2.5 \mathrm{~km})$ depression that is roughly aligned east-west.

\section{F-ring moons}

Prometheus and Pandora orbit inside and outside the F-ring, respectively. The images taken during the Pandora flyby show grooves and debris on the surface of this shepherd moon (Fig. 1E). Although many of the grooves form a pattern concentric to the major axis of the body, there is a

213 slight offset between them, especially noticeable on the sub-Saturn side, which reflects orientations 214 seen in previous observations (21). 
Part of Pandora's leading hemisphere is smooth in comparison to other regions on this moon (Figs. 1E, 3D). The smooth deposits are most continuous near the equator but become patchy at high latitudes, where they appear to be too thin to mute the coarse surface relief along protruding crater rims. The smooth deposits extend approximately $\pm 60^{\circ}$ in latitude, slightly more than the maximum latitude of the ridge on Atlas. This arrangement might indicate the accretion of material, as with the main ring moons. If so, the accretion efficacy on Pandora is at least two orders of magnitude smaller than on Pan and Atlas, and much broader latitudinally. However, variations in resolution, illumination, and viewing geometry make mapping of textural variations on Pandora ambiguous.

\section{Co-orbital moons}

The highest resolution images of the flybys were of Epimetheus, the smaller of the co-orbital moons, reaching scales of 36 and $49 \mathrm{~m} /$ pixel. These data enabled enhanced mapping of grooves and sediment coverings seen in previous observations (23). The grooves are global in occurrence, largely beaded to straight, elongated depressions that appear to be formed in loose regolith. There are some exposures of brighter material apparently devoid of regolith cover (Fig. 1F) that also show elongated lineations, generally slight depressions. These align with the grooves nearby that appear to be regolith features, and largely align with the regolith groove global patterns. This association appears to support a previously-proposed relation of at least some regolith grooves with fractures or other structures in a more rigid underlying bedrock, although the variety of groove morphologies on many objects suggest grooves may have a multiplicity of origins $(23,29,30,31)$.

\section{Colors of the Small Ring Satellites and Pandora}


The whole-disk colors of the ring satellites as measured in ISS broadband filters (32) follow similar trends with distance from Saturn as those found by the VIMS instrument (7-10). The ISS Narrow Angle Camera (NAC) uses paired broadband filters. The CL1:UV3 pair $(0.341 \mu \mathrm{m})$ and CL1:IR3 pair $(0.930 \mu \mathrm{m})$ span the spectral range of the camera, and IR3/UV3 ratios represent the observed brightness value in each CL1:UV3 broadband filter relative to the corresponding value in the CL1:IR3 filter (cf. 6). For reference, Enceladus, the presumed source of ice particles that mute colors on other satellites, has an effectively neutral IR3/UV3 ratio of $1.03 \pm 0.02$ (33).

The spatially resolved colors of Pan, Daphnis, and Atlas can be used to show the effects of material deposited from the rings (supplementary material, Table S2).Closest to Saturn, Pan's average IR3/UV3 ratio is red at $2.5 \pm 0.2$, but significantly smaller than the value of $3.3 \pm 0.2$ of the adjacent A-ring (i.e., Pan is less red than the rings). Farther out, the A-ring IR3/UV3 ratio decreases from $2.7 \pm 0.2$ on the inside of the Keeler gap (which contains Daphnis) to $2.2 \pm 0.3$ on the outside. The mean value is not statistically different from that of Daphnis itself, $2.3 \pm 0.3$. The equatorial ridges on the ring satellites may be very old (4) but the colors most likely reflect a patina of material deposited from geologically recent and ongoing processes. Atlas, which falls just outside the Aring, has an IR3/UV3 ratio $2.4 \pm 0.1$. Pandora, which is near the F-Ring and farther from Saturn, has a lower IR3/UV3 ratio of $1.9 \pm 0$. It lacks an equatorial ridge but possesses smooth deposits which on the leading side extend from the equator to mid-latitudes.

Among the terrains shown in Fig. 3, color differences can be identified in the high-resolution images of all moons except Daphnis, for which the CL1:UV3 images were badly blurred by spacecraft motion. The IR3/UV3 ratio for cratered materials on Pan is about $19 \%$ higher than for its equatorial ridge and reaches approximately the average global value. Similarly, the ratio for 
cratered materials on Atlas is about $16 \%$ higher than for its ridge, but in this case, the global average value closely matches that of Atlas' larger equatorial ridge. For Pandora, the cratered materials have a IR3/UV3 ratio that is $15 \%$ lower than for the smooth materials towards the equator. The global average ratio falls between that of the cratered material and the smooth deposits. Exposed substrate is visible as a scarp on Pan and a bright exposed crater wall on Pandora. On Pan, the IR3/UV3 ratio of exposed substrate is intermediate between the ridge materials and crater materials. However, on Pandora, the corresponding ratio for the exposed crater wall is not statistically distinguishable from that of the cratered material.

\section{Composition}

Compositional information on the surfaces of the moons has been acquired using VIMS (16). Prior to the close flybys of the ring moons, spectra taken by VIMS from greater distances were obtained (7-10). Water ice was the only volatile identified, but the moons' visible colors varied, especially in the $0.35-0.55 \mu \mathrm{m}$ spectral region, which suggested contamination by a reddish chromophore that perhaps came from the ring system itself. This coloring agent is distinct from the low-albedo red material from the Phoebe ring that is deposited on the leading hemisphere of Iapetus and on Hyperion $(7,8)$.

The close flybys of the embedded moons Daphnis and Pan enabled the acquisition of spectra of these moons, although only an IR spectrum (1.0-5.0 $\mu \mathrm{m})$ for Daphnis was successfully obtained. These data provide a test for the origin of the red chromophore in the inner Saturnian system. They also provide rudimentary information on spatial variations in composition on the moon's surfaces, although the spatial resolution is only about 1-2\% (depending on the instrument mode) of ISS's (Supplementary materials, Fig. S1) 
Fig. 4 shows the spectrum of each moon from 0.35-4.2 or 3.6 $\mu \mathrm{m}$ (1-3.6 $\mu \mathrm{m}$ for Daphnis). The only absorption bands detectable are those of water ice at $1.25,1.6,2.0$ and $3.0 \mu \mathrm{m}$. No other volatiles are detectable, including $\mathrm{CO}_{2}$, although its strongest absorption band in this spectral region is at $4.26 \mu \mathrm{m}$, in the noisy region of the spectrum beyond about $3.5 \mu \mathrm{m}$. There is a deep absorption band for crystalline water ice at $1.65 \mu \mathrm{m}$. This spectral band is sensitive to radiation damage (34); its unusual depth compared to Cassini's previously obtained spectra of icy moons (7-10) implies a lack of radiation damage in the ring environment, which is expected given the dearth of high-energy particles in the rings (see below). Water ice spectral bands are also sensitive to grain size, with deeper bands signifying larger grains (35). A larger particle size could signify larger regolith grains in the main ring system than in the E-ring, or it could simply be due to gravitational escape of the smaller particles, some of which could be formed by continual impacts.

In general there is a gradient depending on the position of the moon with respect to the rings, with Pan, which is embedded in the Encke gap, being the reddest and Epimetheus, which is farthest from the rings and closest to Enceladus, being the bluest. (The one exception to this pattern is Pandora's bluer color in the $0.55-0.95 \mu \mathrm{m}$ region.) This effect results from the countervailing processes of contamination by a red chromophore from the main rings and ice particles or water vapor from the E-ring, which originates from Enceladus's plume. 


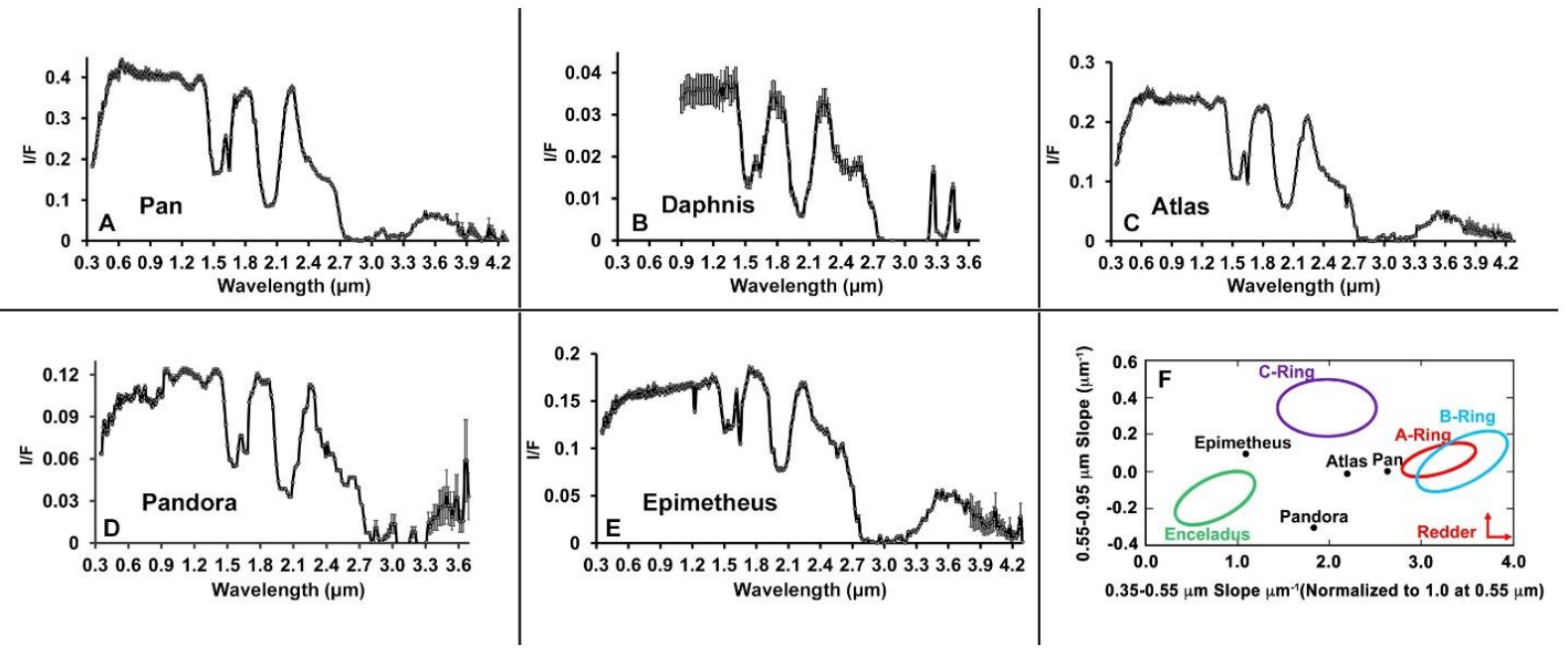

Fig. 4.VIMS Spectra and colors of the five moons and the $\mathbf{A}$ to $\mathbf{C}$ rings. Spectra from 0.35 -

5.1 $\mu \mathrm{m}$ of Pan (A), Daphnis (B), Atlas (C), Pandora (D) and Epimetheus (E) (Noisy data at the long wavelengths are not shown). $\mathrm{I} / \mathrm{F}$ is the reflected intensity compared with the incident solar flux. (F) Color-color plot of Saturn's main ring system and Enceladus $(7,8)$ compared with Epimetheus, Atlas, Pandora, and Pan.

The VIMS colors agree with those derived by ISS above. The VIMS equivalent values at the same wavelengths as the effective wavelengths of the ISS filters yield IR3/UV3 ratios of $2.7 \pm 0.3$ for Pan; $2.2 \pm 0.2$ for Atlas, $1.7 \pm 0.2$ for Pandora, and $1.5 \pm 0.1$ for Epimetheus. (The VIMS spectrum extends to only $0.35 \mu \mathrm{m}$ : the visible slope of the spectra were linearly extrapolated to $0.34 \mu \mathrm{m}$ to match the wavelength of the ISS UV3 filter.) The moons embedded in the rings show spectral differences with the surrounding rings; in general they are less red (Fig. 4F). The VIMS ratio image of Atlas (Fig. S1) shows uniformity between the main body and its equatorial ridge, at least in water ice abundance, which implies the accumulation of particles away from the equator to provide a globally homogeneous surface. Color differences below the spatial resolution of VIMS exist, as detected by ISS in the visible. 
The spectrum of Pan is redder in the 0.35 and $0.55 \mu \mathrm{m}$ region than other Saturnian moons. Atlas, the shepherd moon just outside the A-ring, is also red but less so than Pan, and Pandora, which is associated with the F-ring, even less. The color of Epimetheus is more like that of the mediumsized moons Enceladus and Mimas (7-9). Thus, there is a gradient in color with distance from Saturn's ring system, with the embedded Pan being the reddest. Figure 4A-E shows the slope of the visible spectrum increases as the distance to Saturn increases, and it is quantified in Fig. 4F, which shows the visible colors derived from the flybys with the colors of the main ring system of Saturn (8). These results imply the red chromophore comes from the rings themselves. However, the differences in color between the moons and their adjacent rings - the small moons are consistently bluer than their surrounding rings - could be due to another contaminant: particles of almost pure water ice or vapor from the E-ring. This ring is a diffuse torus that is fed from the plume of Enceladus. The particles have a wide range of orbital elements and predominately impact the leading sides of the main moons (and the trailing side of Mimas), altering their albedos and colors (36-38). The ring moons' leading hemispheres would tend to accrete more fresh grains of water ice than the surrounding ring particles.

The depth of the water ice band at $2.0 \mu \mathrm{m}$ compared to the continuum at $1.8 \mu \mathrm{m}$ (the $1.8 / 2.0 \mu \mathrm{m}$ ratio) is 5.2 \pm 0.1 for Pan, 5.0 \pm 0.2 for Daphnis; $4.4 \pm 0.1$ for Atlas, $3.4 \pm 0.1$ for Pandora, and $2.4 \pm 0.1$ for Epimetheus. The band-depths increase closer to Saturn, most likely due to the increasing particle sizes (35). This is consistent with the moons embedded in the ring (Pan and Daphnis) being coated with main ring particles rather than with smaller particles from the E-ring. (The absorption band at $1.6 \mu \mathrm{m}$ shows a similar but weaker trend). Because the main ring system provides a shield against the E-ring, particle size may be a significant factor in determining the color and reflectivity of these moons. 
Interactions between moons and magnetospheric particles can also alter the moons' colors and albedos $(12,13)$. However, there is a dearth of high energy particles in the vicinity of these moons (see below). Another factor that may alter spectral slopes and band depths is the particle size of the accreted ring particles (35), which may not be the same as that of the native particles.

\section{Ultraviolet and Thermal Infrared Observations of the Moons}

During the Ring-grazing Orbits the spacecraft was in a high radiation and dust environment that produced high background levels in ultraviolet observations with UVIS. The only moon detected was Epimetheus, during the encounter on Feb 21, 2017 (supplementary materials, Fig. S4), in which the signal is only above the background for the longest Far UV wavelengths, $\sim 0.170-0.19$ $\mu \mathrm{m}$. However, this single UV measurement of reflectance places some constraints on surface composition and external effects on Epimetheus. At $72^{\circ}$ solar phase angle (the angle between the spacecraft, Epimetheus, and the Sun), the derived normal reflectance (average over $0.17-0.19 \mu \mathrm{m}$ ) is $0.09 \pm 0.02$. For comparison, this is roughly $1.5-2$ times lower than the reflectance measured at Tethys under similar viewing geometry (39); however, Tethys has a higher visible geometric albedo ( $\sim 1.2$ compared to $\sim 0.73$ for Epimetheus (36)), which indicates that Epimetheus may have a roughly uniformly lower reflectance than Tethys in the UV-visible range. The UV-visible spectral slope and albedo are strongly driven by external effects, because this spectral range senses the uppermost layer of the regolith affected by processes including plasma and E-ring grain bombardment. The UVIS measurement combined with the visible albedo suggests that Epimetheus is not as affected by the brightening effects of the E-ring grains as Tethys is (36), or that there is some other darkening agent or process important at Epimetheus's location. Thus, the UV-visible albedo of Epimetheus may simply reflect the relative importance of the alteration by the reddish lower-albedo chromophore and the icy E-ring particles at this moon's distance. 


\section{A Blackbody Temperature}

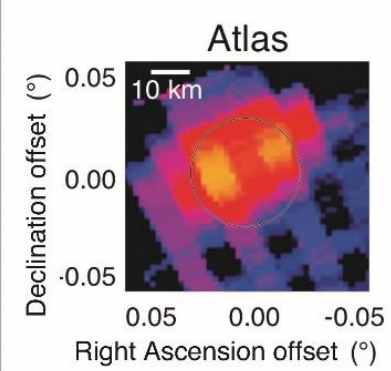

Right Ascension offset $\left({ }^{\circ}\right)$
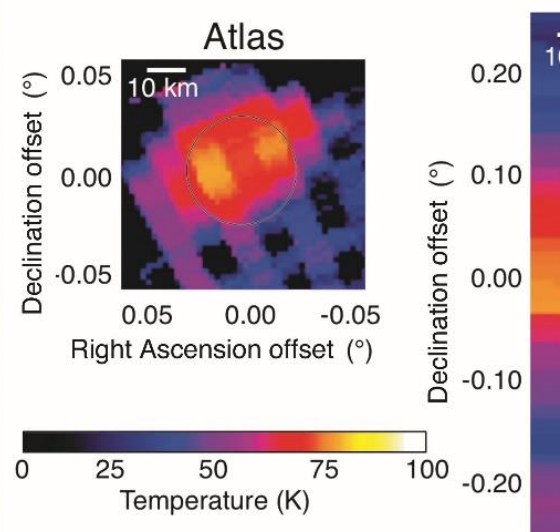
$\overline{10 \mathrm{~km}}$

Epimetheus
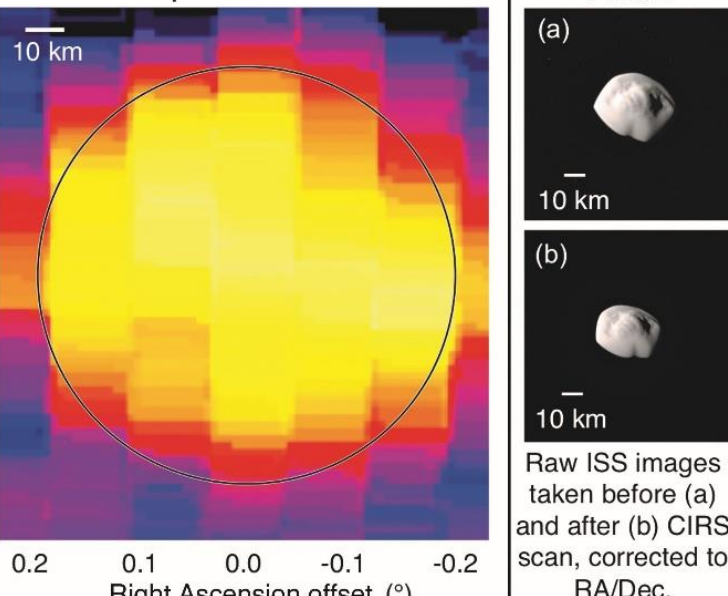

(b)

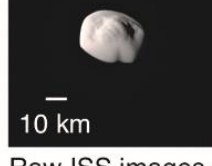

Raw ISS images

taken before (a)

and after (b) CIRS

scan, corrected to

$\mathrm{RA} / \mathrm{Dec}$.

Fig. 5. CIRS thermal infrared and ISS visible light observations of Atlas and Epimetheus. Left:

Panel A: Blackbody temperature distributions of the two moons, determined by fitting a blackbody curve to the full CIRS radiance spectrum at each location. The axes are offsets in Right Ascension and declination, with the origin at the center of the target (note that the IR images do not fall on this location because only the hotter side is detected). Panel B: Right: ISS observations of both targets taken immediately before and after the CIRS scan, on the same orientation (supplementary materials).

\section{Particle Observations}




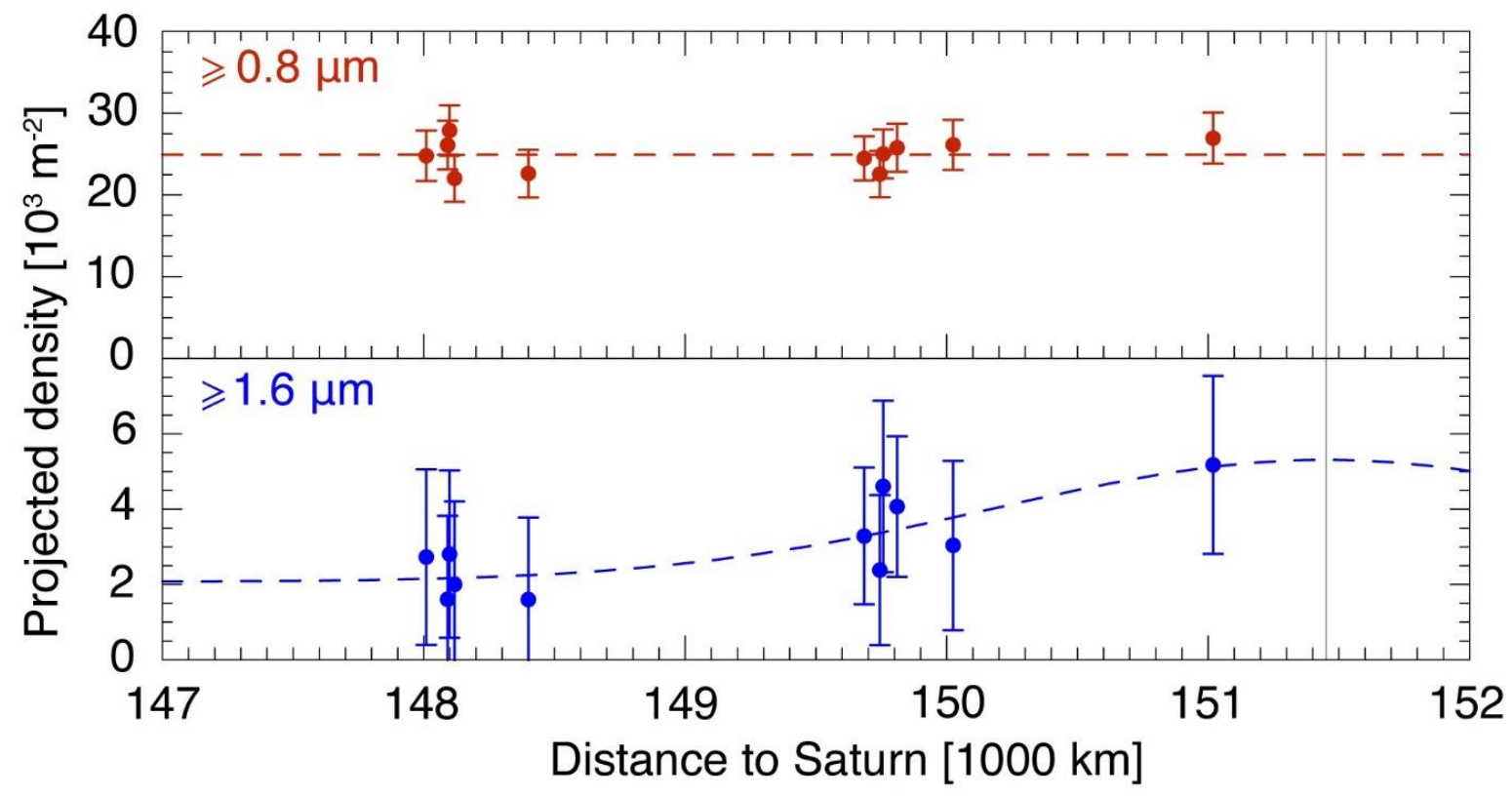

391 
Fig. 6. Radial dust density distribution obtained from CDA-HRD measurements. While the

density of the $\geq 0.8 \mu \mathrm{m}$ sized particles indicates a constant profile (red dashed line), the density of the $\geq 1.6 \mu \mathrm{m}$ sized particles decreases inward from the orbit of Janus and Epimetheus (vertical gray line). The dust distribution of the larger particles is modeled by a Gaussian distribution (blue dashed line) with a maximum at the mean radial position of Janus and Epimetheus, plus a constant background density. Error bars are based on Poisson statistics.

Many dust rings are formed by ejecta from high-velocity impacts of interplanetary micrometeoroids eroding the surfaces of satellites without atmospheres. The measured particle number in the Janus-Epimetheus ring constrains the poorly known parameters of the impact-ejection dust creation model $(41,42)$ at Saturn. Using an unfocussed flux of $>3.6 \cdot 10^{-16} \mathrm{~kg} \mathrm{~m}^{-2} \mathrm{~s}^{-1}$ with a mean impact speed of $4.3 \mathrm{~km} \mathrm{~s}^{-1}$ (43), the dust production rate from both moons totals about $0.81 \mathrm{~kg} \mathrm{~s}$ ${ }^{1}\left(0.57 \mathrm{~kg} \mathrm{~s}^{-1}\right.$ from Janus and $0.24 \mathrm{~kg} \mathrm{~s}^{-1}$ from Epimetheus). This corresponds to $9.1 \cdot 10^{11}$ particles larger than $1.6 \mu \mathrm{m}$ per second $\left(6.4 \cdot 10^{11} \mathrm{~s}^{-1}\right.$ from Janus and 2.7 $100^{11} \mathrm{~s}^{-1}$ from Epimetheus) assuming a cumulative power law size distribution for a dust diameter $\mathrm{d} \propto \mathrm{d}^{-\alpha}$ with $\alpha=2.4$ and a maximal ejecta mass of $1 \cdot 10^{-8} \mathrm{~kg}$, consistent with observations of impact-generated dust clouds around the Galilean moons $(44,41)$.

To explain the measured number of ring particles, this comparably high production rate requires a shallow slope of the cumulative ejecta velocity $\mathrm{v}$ distribution $\propto \mathrm{v}^{-\gamma}(\gamma=1)$, and a kinetic energy dissipation at the higher end of the values predicted by laboratory experiments $(45,46)$. The kinetic energy ratio of ejecta to impactor is $5 \%$.) This points to a highly dissipative and porous (snow or 
regolith) surface. We find that most ejecta are gravitationally bound to the moons and fall back to their surface, while only about $5 \%$ of them for Janus and $7 \%$ for Epimetheus escape to the surrounding ring. Numerical simulations (supplementary material, text) show that most of the ring particles are recaptured by the source moons, after an average lifetime of 60 years, resulting in an estimate of $9.8 \cdot 10^{19}$ ring particles larger than $1.6 \mu \mathrm{m}$. This value is in rough agreement with the observed value of $2 \pm 1 \cdot 10^{19}$, which in turn constrains the poorly known parameters of the impactejection model, which can vary by orders of magnitude. The CDA Chemical Analyzer (8) recorded mass spectra of submicrometer-sized dust particles $(0.1 \mu \mathrm{m}-0.4 \mu \mathrm{m})$. The compositional analysis of these spectra recorded near the ring plane shows mostly ice grains but also about 3 percent pure silicate grains or ice-silicate mixtures (supplementary material, Fig. S5).The source of the icy particles could either be the inner edge of the E-ring or surface ejecta of the nearby small ice moons. Because silicate-rich grains of this size have not been detected in the E-ring (47), these must originate from a different source, possibly the nearby moons Janus and Epimetheus or the Fand G-rings.

The Low Energy Magnetospheric Measurements System (LEMMS) of the MIMI energetic charged particle detector surveyed the planet's radiation belts inward of Saturn's G-ring and monitored the energetic particle environment of the five small moons. LEMMS measures energetic electrons and ions above 18 and $27 \mathrm{keV}$ respectively, reaching into the $\mathrm{MeV}$ energy range. The region inward of Saturn's G-ring has been sampled in the past on several occasions with Pioneer 11 and Cassini (48-50). It contains the location where both Saturn's proton and electron radiation belts have their highest intensities, which lies between the G-ring and Janus and Epimetheus's orbits. Inward of that maximum, intensities drop gradually up to the outer edge of Saturn's A-ring 
which absorbs all energetic particles. Superimposed on the radial profile of radiation belt fluxes are localized dropouts originating from Saturn's moons and rings (51). While several of these features can be attributed to specific moons, like Janus and Epimetheus (52), any influences by Pandora, Prometheus and Atlas (orbiting within the radiation belt boundaries) are less clear. These moons orbit close to Saturn's A and F-rings, complicating the separation of the different contributions. Understanding how effectively these moons sweep-out particle radiation also determines the radiation environment which their surfaces are exposed to.

Fig. 7A shows count-rates of $>12$ and $>25 \mathrm{MeV}$ protons as a function of L-shell (L), averaged over the Proximal Orbits. The L-shell is defined as the distance from Saturn that a magnetic field line intersects the magnetic equator, expressed as multiples of the planet's radius (Saturn's radius is $60268 \mathrm{~km})$. The spacecraft L-shell at the time of data collection is determined by mapping along Saturn's magnetic field using a third-order multipole model for Saturn's internal magnetic field (53). Fig. 7 shows the previously established sectorization of the $\mathrm{MeV}$ proton radiation belts, due to the moons and rings that absorb any protons diffusing across their orbits $(54,55)$. Among these different sectors, the least well-characterized by previous observations is the "Minor Belt", centered at approximately $\mathrm{L}=2.29$. The gap immediately outside the Minor Belt is centered near the F-ring ( $\mathrm{L} \sim 2.32$ ); we find that the gap boundaries coincide with the L-shells of Prometheus and Pandora (Fig. 7A). Pandora and Prometheus are therefore absorbing protons at a rate that is high enough to counter the diffusive influx of protons from the surrounding belt sectors. Effectively, the two moons and the F-ring form an extended obstacle to proton radiation. The net result is that the weathering of Pandora's and Prometheus's surfaces by energetic protons is negligible since they orbit within the proton radiation gaps they create. Atlas's effects cannot be distinguished from 
460 those of the A-ring, but that moon is also exposed to very low proton fluxes. Overall, almost all of 461 Saturn's inner moons (except Dione, Rhea or minor moons like Anthe or Pallene) orbit in regions 462 free from energetic ions (56-58). This is unlike Jupiter's satellites, whose surface chemistry and 463 thin atmospheric properties are strongly affected by irradiation from high fluxes of $\mathrm{keV}$ and $\mathrm{MeV}$ 464 particles $(59,60)$. 

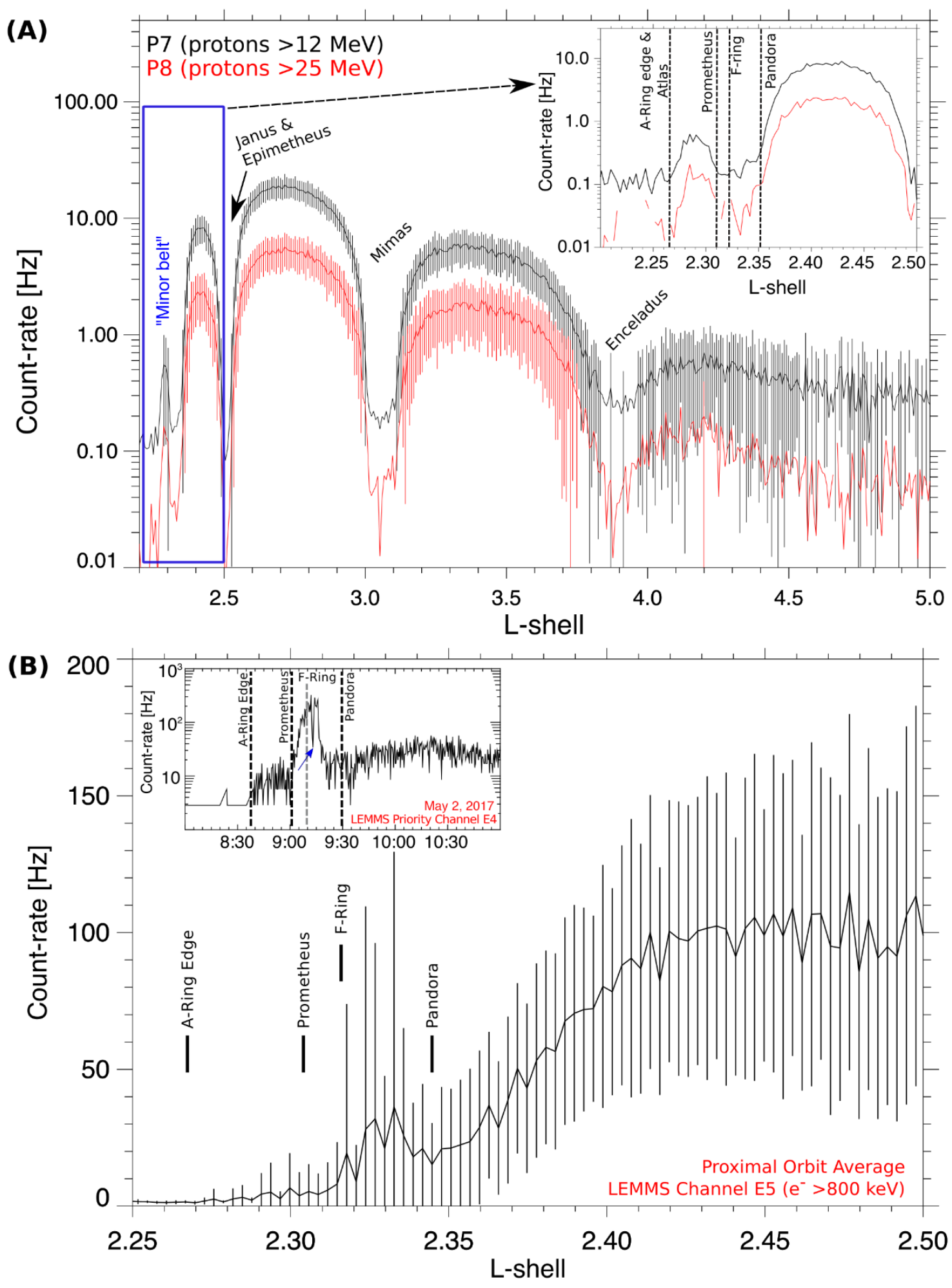
Fig. 7. Average count-rates for protons (A) and electrons(B), measured by MIMI/LEMMS. The channels for protons are $>12$ and $>25 \mathrm{MeV}$, and $>800 \mathrm{keV}$ for electrons; both sets of data are shown as a function of L-shell, with 1- $\sigma$ error bars. Absence of error bars indicates an uncertainty larger than the corresponding mean value. The orbits of several of Saturn's large icy moons are also marked. The inset in (A) zooms into the region of the Minor Belt, highlighting the absorbing effects of Atlas, Pandora, Prometheus and the A- and F-rings. The inset in (B) shows a high time resolution series of observations (1 sample per $0.3125 \mathrm{sec}$ ) from LEMMS obtained during the second proximal orbit, on May 2, 2017. The blue arrow marks an electron microsignature within one of the MeV electron spikes seen consistently during Cassini's outbound crossings near the Lshell of the A-ring's outer edge.

Fig. 7B shows the Proximal Orbit averages of electron count-rates from LEMMS channel E5 $(>0.8$ $\mathrm{MeV}$ ) as a function of L-shell. Electron radiation levels are more variable than those of protons, as the large error bars indicate, because moons and rings are not effective in sweeping out electrons from their orbits $(51,61)$. Inside $\mathrm{L}=2.4$ (inwards of the Janus and Epimetheus orbits) electron rates fall slowly towards the outer edge of the A-ring $(\mathrm{L}=2.27)$. This drop is interrupted by an enhancement of the mean electron rates, near the L-shells of the F-ring, Pandora and Prometheus. In the absence of an $\mathrm{MeV}$ electron source, such an enhancement, which was absent from past observations at the same L-shell $(52,62)$, is unexpected. The $1-\sigma$ error bars in that location span more than two orders of magnitude in amplitude, indicating much higher variability than in the surrounding regions. This large scatter is attributed to spikes of enhanced $\mathrm{MeV}$ electron flux observed in 18 out of the 22 outbound crossings outwards of the A-ring's edge and between $\mathrm{L}=2.31$ and $\mathrm{L}=2.35$. The radial extent of an individual spike is less than $1800 \mathrm{~km}$ along the equatorial 
plane, and the electron intensity within them can be enhanced by as much as a factor of 300 compared their surroundings. The inset of Fig. 7B shows one such resolved spike, captured by the high time resolution measurements of LEMMS Priority channel E4 (0.8-4.2 MeV) on May 2, 2017. Because most measurements in the inbound portion of Cassini's orbit showed no evidence of similar spikes in the same L-shell range, we deduce that these features are usually located a few hours after local noon, and their longitudinal extent ranges between $22^{\circ}$ and $37^{\circ}$ in the clockwise direction, starting from a magnetospheric local time of 13:20. The longitudinal extent cannot be constrained in the anticlockwise direction. Most of these enhancements were seen around the Lshells of the F-ring, Prometheus and Pandora. This electron belt component is therefore limited in local-time range. As a result, energetic electron bombardment of the three moons is variable in intensity, episodic and occurs only for a fraction of their orbit around Saturn. Material interaction signatures of energetic electrons are seen as localized depletions (microsignatures) within the electron spikes. These may be due to Atlas, Prometheus, Pandora or F-ring clumps (62); an example is shown in the Inset of Fig. 7B and could have formed only after the electron enhancement developed.

There is no discernible signal of trapped electron or proton radiation at the orbits of the Keeler and Encke gaps, where Daphnis and Pan are orbiting (53).

\section{Summary and Conclusions}

The low densities of the small moons of Saturn, measured during the flybys, are consistent with a multi-stage formation scenario involving accretion of ring material $(4,5)$. The color of the moons embedded in the A-ring are more consistent with the rings the closer the moons are to Saturn. This suggests there is an ongoing accretion of a reddish chromophore that be a mixture of organics and 
iron $(8-11)$, onto the surfaces of the moons. The difference in color between the moons and their adjacent ring may be explained by the accretion of bright, icy particles or, more likely, water vapor from the E-ring. Each moon's surface is subjected to a balance between these two ongoing processes, with their distance from Saturn and Enceladus determining the result color, as illustrated in Fig. 4F. The detection of abundant ice grains by CDA supports this view. The bluer core of Atlas is also explained by the accretion of E-ring particles, which have a wider range of inclinations than main ring particles. If the ring moons formed from the same material as the rings, they would have been the same color, and the color gradient may be solely due to contamination by the Ering. The size of particles on the moons' surfaces also plays a role, especially for the moons embedded in the main ring system, which would shield these moons from the E-ring.

The dearth of high-energy ions close to the moons lessens the alteration processes caused by bombardment with magnetospheric particles. The strong crystalline water ice band at $1.65 \mu \mathrm{m}$ also suggests low radiation damage. This low energy plasma environment is unlike the main moons of Saturn, especially Dione and Rhea, as they dwell in a region where alterations by ions is substantial Particle radiation would tend to darken and redden the surfaces, so the red chromophore on the trailing hemispheres of the main moons may be unrelated to the red material contributing to the colors of the ring moons (63). Contamination of Saturn's rings by bright icy particles or water vapor offers counterevidence to previous arguments that the observed brightness of the rings indicates recent formation (64).

The moons' geology records a complex history, including groove formation caused by tidal stresses and accretion of ring particles. The CDA finding of a porous surface further supports substantial accretion. Although the topography and surface slopes strongly suggest the equatorial ridges of Pan and Atlas are accreted from the rings and are not formed by normal surface transport, 
there is variety of forms of ridges on these objects. The flyby images strongly suggest exposures of a solid substrate distinct from the mobile regolith that covers many small Solar System objects.

\section{Acknowledgements}

The authors are grateful to the Cassini project engineers and staff for their dedicated service that led to the success of the final stages of the mission.

\section{Funding}

This paper was funded by the Cassini Project. Part of this research was carried out at the Jet Propulsion Laboratory, California Institute of Technology under contract to the National Aeronautics and Space Administration. MSeiss, RS, HH, MSachse, FS, TA, SK, MKhawaja, GMK, FP and JSimolka were supported by the Deutsches Zentrum für Luft-und Raumfahrt (OH 1401 and 1503) and by the Deutsche Forschungsgemeinschaft (Ho5720/1-1). GF and MC were supported by the Italian Space Agency.

\section{Author contributions}

BJB, PCT, ER, CH, MS, ARH, PH, HH, NKhawaja, SH, TWM: planning observations, data analysis and writing; RHB, RS, TA, KHB, SK, SMK, DM, GM-K, PDN, CCP, HR, JSimolka, LAS: instrument development and planning observations; RNC, TD, MS, FS, JSpencer, NKrupp, FP, CP, GHJ, PK, JL: data analysis and planning observations; GF, MC, TE: data analysis.

\section{Competing Interests}

None.

\section{Data and materials availability}

All data used in this paper are archived in NASA's Planetary Data System (PDS). The ISS, VIMS, CIRS, and UVIS data can be found at: https://pds-rings.seti.org/cassini/. 
The periods of data acquisition in Universal Time are Pan: March 7, 2017 16:35-19:05; Daphnis: January 16, 2017 11:33-14:03; Atlas: April 12, 2017 11:30-14:10; Pandora: December 18, 2016 19:59-21:54; Epimetheus: January 30, 2017 19:22-21:12, and February 21, 2017 09:33-10:43.

The CDA and MIMI data were acquired continuously throughout the F-ring orbital period, lasting from November 30, 2016 to April 22, 2017 and during the Proximal Orbits, which lasted from the end of the F-ring orbits until the end of mission on September 15, 2017.

CDA observations can be found at: https://pds.nasa.gov/ds-view/pds/viewDataset.jsp?dsid=COD-CDA-3/4/5-DUST-V1.0, and MIMI data can be found at:

\section{https://pds.nasa.gov/ds-view/pds/viewDataset.jsp?dsid=CO-S-MIMI-4-LEMMS-CALIB-V1.0}

Pan gravity data is included in the supplementary materials.

The software for the CDA modeling in the supplementary materials can be found at

\section{References}

1. C. C. Porco et al., Cassini imaging science: Initial results on Saturn's rings and small satellites. Science 307, 1237-1242 (2005).

2. M. R. Showalter, Visual detection of 1981S13, Saturn's eighteenth satellite, and its role in the Encke gap. Nature 351, 709-713 (1991).

3. B. A. Smith et al., Encounter with Saturn - Voyager 1 imaging science results. Science 212, 163-191 (1981).

4. C. C. Porco, P. C. Thomas, J. W. Weiss, D. C. Richardson, Saturn's small inner satellites:

Clues to their origins. Science 318, 1602 (2007).

5. S. Charnoz, A. Brahic, P. C. Thomas, C. C. Porco, The equatorial ridges of Pan and Atlas: Terminal accretionary ornaments? Science 318, 1622-1624 (2007).

6. P. C. Thomas et al. The inner small satellites of Saturn: A variety of worlds. Icarus 226, 9991019 (2013). 
584

585

586

587

588

589

590

591

592

593

594

595

596

597

598

599

600

601

602

603

604

7. G. Filacchione et al., Saturn's icy satellites investigated by Cassini-VIMS. II. Results at the end of nominal mission. Icarus 206, 507-523 (2010).

8. G. Filacchione et al., Saturn's icy satellites and rings investigated by Cassini-VIMS: III Radial compositional variability. Icarus 220, 1064-1096 (2012).

9. G. Filacchione, et al., The radial distribution of water ice and chromophores across Saturn's system. Astrophys. J. 766, 76-80 (2013).

10. B. J. Buratti et al., Cassini spectra and photometry $0.25-5.1 \mu \mathrm{m}$ of the small inner satellites of Saturn. Icarus 206, 524-536 (2010).

11. R. N. Clark et al., The surface composition of Iapetus: Mapping results from Cassini VIMS. Icarus 218, 831-860 (2012).

12. P. Schenk et al., Plasma, plumes and rings: Saturn system dynamics as recorded in global color patterns on its midsize icy satellites. Icarus 211, 740-757 (2011).

13. A. R. Hendrix, G. Filacchione, C. Paranicas, P. Schenk, F. Scipioni, Icy Saturnian satellites:

Disk-integrated UV-IR characteristics and links to exogenic processes. Icarus 300, 103-114 (2018).

14. T . V. Johnson, J. I. Lunine, Saturn's moon Phoebe as a captured body from the outer Solar System. Nature 435, 69-71 (2005).

15. C. C. Porco et al., Cassini maging science: Instrument characteristics and anticipated scientific investigations at Saturn. Space Sci. Revs. 115, 363-497 (2004).

16. R. H. Brown et al., The Cassini Visual and Infrared Mapping Spectrometer (VIMS) investigation. Space Sci. Res. 115, 111-118 (2004). 
605

606

607 608

609

610

611

612

613

614

615

616

617

618

619

620

621

622

623

624

17. F. M. Flasar et al., Exploring the Saturn system in the thermal infrared: The composite infrared spectrometer. Space Sci. Res. 115, 169-297 (2004).

18. L. W. Esposito et al., The Cassini ultraviolet imaging spectrograph investigation. Space Sci. Res. 115 299-361 (2004).

19. R. Srama et al., The Cassini cosmic dust analyzer. Space Sci. Res. 114, 465-518 (2004).

20. S. M. Krimigis et al., Magnetosphere IMaging Instrument (MIMI) on the Cassini mission to Saturn/Titan. Space Sci. Res. 114, 233-329 (2004).

21. M. M. Hedman et al. Three tenuous rings/arcs for three tiny moons. Icarus 199, 378-386 (2009).

22. M. M. Hedman et al Aegaeon (Saturn LIII), a G-ring object. Icarus 207, 433-446 (2010).

23. S. J. Morrison, P. C. Thomas, M. S. Tiscareno, J. A. Burns, J. Veverka, Grooves on small Saturnian satellites and other objects: Characteristics and significance. Icarus 204, 262-270 (2009).

24. M. S. Tiscareno, P. C. Thomas, J. A. Burns, The rotation of Janus and Epimetheus. Icarus 204, 254-261 (2009).

25. N. J. Cooper, S. Renner, C. D. Murray, M. W. Evans, Saturn's inner satellites: Orbits, masses, and the chaotic motion of Atlas from new Cassini imaging observations. Astron. J. 149, $27-45(2015)$

26. J. W. Weiss, C. C. Porco, M. S. Tiscareno, Ring edge waves and the masses of nearby satellites. Astron. J. 138, 272-286 (2009). 
28. A. W. Harris, E. G. Fahnestock, P. Pravec, On the shapes and spins of "rubble pile" asteroids. Icarus 199, 310-318 (2009).

29. S. J. Weidenschilling, A possible origin for the grooves of PHOBOS. Nature 282, 697 (1979).

30. D. L. Buczkowski, O. S. Barnouin-Jha, L. M. Prockter, 433 Eros lineaments: Global mapping and analysis. Icarus 193, 39-52 (2008).

31. M. Nayak, E. Asphaug, Sesquinary catenae on the martian satellite Phobos from reaccretion of escaping ejecta. Nature Communications. 7, 12591 (2016).

32. R. A.West et al., In-flight calibration of the Cassini imaging science sub-system cameras. Planet. and Space Sci. 58, 1475-1488 (2010).

33. P. C. Thomas, M. Tiscareno, P. Helfenstein, "The Inner Small Satellites of Saturn and Hyperion." in Enceladus and the Icy Moons of Saturn, P. Schenk, R.Clark, C. Howett, A. Verbiscer, H. Waite, Eds. (University of Arizona, 2018).

34. R. M. E. Mastrapa, R. H. Brown, Ion irradiation of crystalline H2O-ice: Effect on the 1.65$\mu \mathrm{m}$ band. Icarus 183, 207-214 (2006).

35. R. N. Clark, Water frost and ice - The near-infrared spectral reflectance 0.65-2.5 microns. $J$ Geophys. Res. 86, 3087-3096 (1981).

36. D. P. Hamilton, J. A. Burns, Origin of Saturn's E-ring: Self-sustained, naturally. Science 264, 550-553 (1994). 
37. B. J. Buratti, J. A. Mosher, T. V. Johnson, Albedo and color maps of the Saturnian satellites. Icarus 87, 339-357 (1990).

38. A. Verbiscer, R. French, M. Showalter, P. Helfenstein, Enceladus: Cosmic graffiti artist caught in the act. Science 315, 815 (2007).

39. E. M. Royer, Hendrix, A., First far-ultraviolet disk-integrated phase curve analysis of Mimas, Tethys and Dione from the Cassini-UVIS data sets. Icarus 242. 158-171 (2014).

40. C. C. Porco, Rings of Saturn (R/2006 S 1, R/2006 S 2, R/2006 S 3, R/2006 S 4). IAU Circ., No. 8759, \#1 (2006).

41. A. V. Krivov, M. Sremečvić, F. Spahn, V. V. Dikarev, K. V. Kholshevnikov, Impactgenerated dust clouds around planetary satellites: spherically symmetric case. Planetary and Space Science 51, 251-269 (2003).

42. F. Spahn et al., E-ring dust sources: Implications from Cassini's dust measurements. Planet. and Space Science 54, 1024-1032 (2006).

43. N. Altobelli, S. Kempf, F. Postberg, A. Poppe, C. Fischer, T. Albin, R. Srama, Exogenous Dust at Saturn. Cassini Science Symposium (Boulder), http://lasp.colorado.edu/media/projects/cassini/meetings/css2018/2-TUESDAY/3-Rings_3 (R3-

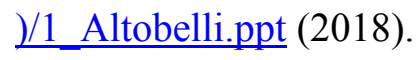

44. H. Krüger, A. V. Krivov, M. Sremčević, E. Grün, Impact-generated dust clouds surrounding the Galilean moons. Icarus 164, 170-187 (2003).

45. N. Asada, Fine fragments in high-velocity impact experiments. J Geophys. Res. 90, 12,445$12,453(1985)$ 
667

668

669

670

671

672

673

674

675

676

677

678

679

680

681

682

683

684

685

686

687

46.W. K. Hartmann, Impact experiments. I - Ejecta velocity distributions and related results from regolith targets. Icarus $\mathbf{6 3}, 69-98$ (1985).

47. J. K. Hillier et al., Interplanetary dust detected by the Cassini CDA Chemical Analyser. Icarus 190, 643-654 (2007).

48. J. A. van Allen et al., Pioneer 11 observations of energetic particles in the Jovian magnetosphere. Science 188, 459-462 (1975).

49. F. B. McDonald, A. W. Schardt, J. H. Trainor, If you've seen one magnetosphere, you haven't seen them all - Energetic particle observations in the Saturn magnetosphere. J. Geophys. Res. 85, 5813-5830 (1980).

50. S. M. Krimigis et al., Dynamics of Saturn's magnetosphere from MIMI during Cassini's orbital insertion. Science 307, 1270-1273 (2005).

51. E. Roussos et al., Evidence for dust-driven, radial plasma transport in Saturn's inner radiation belts. Icarus 274, 272-283 (2016).

52. J. A. van Allen, Findings on rings and inner satellites of Saturn of Pioneer 11. Icarus 51, 509-527 (1982).

53. E. Roussos, et al., Sources, sinks and transport of energetic electrons near Saturn's main rings, Geophys. Res. Lett. 45 (2018). https://doi.org/10.1029/2018GL078097

54. E. Roussos et al., Discovery of a transient radiation belt at Saturn. Geophys. Res. Lett. 35, $22106(2008)$.

55. P. Kollmann, E. Roussos, A. Kotova, C. Paranicas, N. Krupp, The evolution of Saturn's radiation belts modulated by changes in radial diffusion. Nature Astronomy. 1, 872-877 (2017). 
688

689

690

691

692

693

694

695

696

697

698

699

700

701

702

703

704

705

706

707

56. B. D. Teolis et al., Cassini finds an oxygen-carbon dioxide atmosphere at Saturn's icy moon Rhea. Science 330, 1813 (2010).

57. P. Kollmann et al., Energet60ic particle phase space densities at Saturn: Cassini observations and interpretations. J. Geophys. Res. (Space Physics) 116, A05222 (2011).

58. C. Paranicas et al., Energetic charged particle weathering of Saturn's inner satellites. Planet. Space Sci. 61, 60-65 (2012).

59. B. H. Mauk et al., Energetic ion characteristics and neutral gas interactions in Jupiter's magnetosphere. Journal Geophys. Res. (Space Physics) 109, A09S12 (2004).

60. J. F. Cooper, R. E. Johnson, B. H. Mauk, H. B. Garrett, N. Gehrels, Energetic Ion and Electron Irradiation of the Icy Galilean Satellites. Icarus 149, 133-159 (2001).

61. E. Roussos et al., The variable extension of Saturn's electron radiation belts. Planet. Space Sci. 104, 3-17 (2014).

62. J. N. Cuzzi, J. A. Burns, Charged particle depletion surrounding Saturn's F ring - Evidence for a moonlet belt? Icarus 74, 284-324 (1988).

63. R. E. Johnson, T. I. Quickenden, Photolysis and radiolysis of water ice on outer solar system bodies. J. Geophys. Res. 102, 10985-10996 (1997).

64. Z. Zhang et al., Exposure age of Saturn's A and B rings, and the Cassini Division as suggested by their non-icy material content. Icarus 294, 14-42 (2017). 65. C. H. Acton, Ancillary data services of NASA's Navigation and Ancillary Information Facility. Planetary and Space Science 44, 65-70 (1996). 
66. C. H. Acton, N. Bachman, B. Semenov, E. Wright, A look towards the future in the handling of space science mission geometry. Planetary and Space Science 150, 9-12 (2018).

67. S. Jurac, R. E. Johnson, J. D. Richardson, Saturn's E Ring and Production of the Neutral Torus. Icarus 149, 384-396 (2001).

68. M. K. Elrod, W.-L. Tseng, A. K. Woodson, R. E. Johnson, Seasonal and radial trends in Saturn's thermal plasma between the main rings and Enceladus. Icarus 242, 130-137 (2014).

69. M. Horányi, A. Juhász, G. E. Morfill,, Large-scale structure of Saturn's E-ring.Geophysical Research Letters 35, L04203 (2008).

70. X. Liu, M. Sachse, F. Spahn, J. Schmidt, Dynamics and distribution of Jovian dust ejected from the Galilean satellites. Journal of Geophysical Research (Planets) 121, 1141-1173 (2016).

71. M. Horányi, J. A. Burns, M. M. Hedman, G. H. Jones, S. Kempf, "Diffuse Rings" in Saturn from Cassini-Huygens, M. K. Dougherty, L. W. Esposito, S. M. Krimigis, eds. (Springer, 2009), 511-536.

72. M. E. Burton, M. K. Dougherty, C. T. Russell, Model of Saturn's internal planetary magnetic field based on Cassini observations. Planetary and Space Science 57, 1706-1713 (2009).

73. R. A. Jacobson, et al., The Gravity Field of the Saturnian System from Satellite Observations and Spacecraft Tracking Data. Astronomical Journal 132, 2520-2526 (2006).

74. D. Koschny, E. Grün, Impacts into Ice-Silicate Mixtures: Ejecta Mass and Size Distributions. Icarus 154, 402-411 (2001).

75. G. Colombo, D. A. Lautman, I. I. Shapiro, The Earth's Dust Belt: Fact or Fiction?, 3, Lunar Ejecta. Journal of Geophysical Research 71, 5719-5731 (1966).

76. A. Krivov, A. Jurewicz, The ethereal dust envelopes of the Martian moons. Planetary and Space Science 47, 45-56 (1998). 
731

732

733

734

735

736

737

77. M. Horányi, et al., A permanent, asymmetric dust cloud around the Moon. Nature 522, 324$326(2015)$.

78. M. Sachse, A planetary dust ring generated by impact-ejection from the Galilean satellites. Icarus 303, 166-180 (2018).

79. K. R. Housen, K. A. Holsapple, Ejecta from impact craters. Icarus 211, 856-875 (2011).

80. H. Krüger, A. V. Krivov, E. Grün, A dust cloud of Ganymede maintained by hypervelocity impacts of interplanetary micrometeoroids. Planetary and Space Science 48, 1457-1471 (2000).

81. A. V. Krivov, H. Krüger, E. Grün, K.-U. Thiessenhusen, D. P. Hamilton, A tenuous dust ring of Jupiter formed by escaping ejecta from the Galilean satellites. Journal of Geophysical Research (Planets) 107, 5002 (2002).

82. H. Hoffmann, T. Moldenhawer, T. Münch, J. Schmidt, M. Seiler, F. Spahn (2019). https://doi.org/10.5281/zenodo.2572758

83. F. Postberg et al., Sodium salts in E-ring ice grains from an ocean below the surface of Enceladus. Nature 459, 1098-1101 (2009)

84. K. Fiege et al., Calibration of relative sensitivity factors for impact ionization detectors with high-velocity silicate microparticles. Icarus 241, 336-345 (2014).

(1)

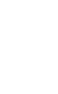

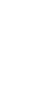

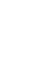


Table 1: Summary of the close flybys of Saturn's ring moons during the Ring-grazing 760

\section{Orbits}

\begin{tabular}{|l|l|l|l|l|l|l|}
\hline Moon & $\begin{array}{l}\text { Semi-major } \\
\text { axis }\left(\mathrm{R}_{\mathrm{s}}\right)\end{array}$ & $\begin{array}{l}\text { Rotation } \\
\text { rate (days) }\end{array}$ & $\begin{array}{l}\text { Date of } \\
\text { flyby }\end{array}$ & $\begin{array}{l}\text { Closest } \\
\text { approach } \\
(\mathrm{km})\end{array}$ & $\begin{array}{l}\text { Best } \\
\text { image } \\
\text { resolution } \\
\text { (m/pixel) }\end{array}$ \\
\hline Pan & 2.22 & 0.575 & $\begin{array}{l}7 \text { March } \\
2017\end{array}$ & $\mathbf{2 2 , 2 4 7}$ & & 147 \\
\hline Daphnis & 2.26 & 0.594 & 16 Jan 2017 & $\mathbf{2 2 , 3 3 6}$ & & 170 \\
\hline Atlas & 2.29 & 0.602 & $\begin{array}{l}12 \text { April } \\
2017\end{array}$ & $\mathbf{1 0 , 8 4 8}$ & & 76 \\
\hline Pandora & 2.35 & 0.629 & $\begin{array}{l}18 \text { Dec } \\
2016\end{array}$ & $\mathbf{2 2 , 1 5 7}$ & & 132 \\
\hline Epimetheus & 2.51 & 0.695 & 30 Jan 2017 & $\mathbf{3 6 2 5}$ & & 36 \\
\hline Epimetheus & 2.51 & 0.695 & $\begin{array}{l}21 \text { Feb } \\
2017\end{array}$ & $\mathbf{8 2 6 6}$ & & 82 \\
\hline
\end{tabular}

Materials and methods

766 Supplementary text

767 Figs. S1 to S5

768 Tables S1 to S4. 
771 


\section{Science \\ IIAAAS}

\section{Supplementary Materials for}

Close Cassini Flybys of Saturn's Ring Moons Pan, Daphnis, Atlas, Pandora, and Epimetheus

B. J. Buratti, P. C. Thomas, E. Roussos, C. Howett, M. Seiß, A. R. Hendrix, P. Helfenstein, R. H. Brown, R. N. Clark, T. Denk, G. Filacchione, H. Hoffmann, G. H. Jones, N. Khawaja, P. Kollmann, N. Krupp, J. Lunine, T. W. Momary, C. Paranicas, F. Postberg, M. Sachse, F. Spahn, J. Spencer, R. Srama, T. Albin, K. H. Baines, M. Ciarniello, T. Economou, S. Hsu, S. Kempf, S. M. Krimigis, D. Mitchell, G. Moragas-

Klostermeyer, P. D. Nicholson, C. C. Porco, H. Rosenberg, J. Simolka, L. A. Soderblom

correspondence to: Bonnie.Buratti@jpl.nasa.gov

This PDF file includes:

Materials and Methods

Supplementary Text

Figs. S1 to S5

Tables S1 to S4 


\section{Materials and Methods}

\section{Visible Infrared Mapping Spectrometer Observations}

The wavelength range of VIMS, from $0.35 \mu \mathrm{m}$ to $5.1 \mu \mathrm{m}$, covers $99 \%$ of the reflected solar spectrum in 352 spectral channels, with spatial resolution of 0.5 mradian and spectral resolution ranging from $1.46 \mathrm{~nm}$ in the visible region $(0.35-1.05 \mu \mathrm{m})$ to $16.6 \mathrm{~nm}$ in the Near IR $(0.85-5.1$ $\mu \mathrm{m})$. These are key spectral ranges for identifying volatiles including water ice, organics, and minerals. VIMS was also capable of a high-resolution spatial mode offering double resolution in one dimension. The instrument had separate visible and infrared channels, with visible light captured by a 512 by 512 CCD detector and IR photons captured on a 1 by 256 InSb detector.

Fig. S1 shows the best images for the five moons at 1.38 (1.48 for Pan), 2.01, and $3.50 \mu \mathrm{m}$ (only 2.01 is shown for Daphnis, due to the low spatial resolution of the images; a positive identification was made by coaligning the VIMS and ISS images). A ratio image of $1.76 / 2.01 \mu \mathrm{m}$, representing the spectral continuum to the most prominent water ice band, is also shown. No spatial variations in the water icy band imply uniformity in abundance and texture on the individual moons. Due to its much higher spatial resolution, ISS is better suited to seeking visible color variations on the moons. 


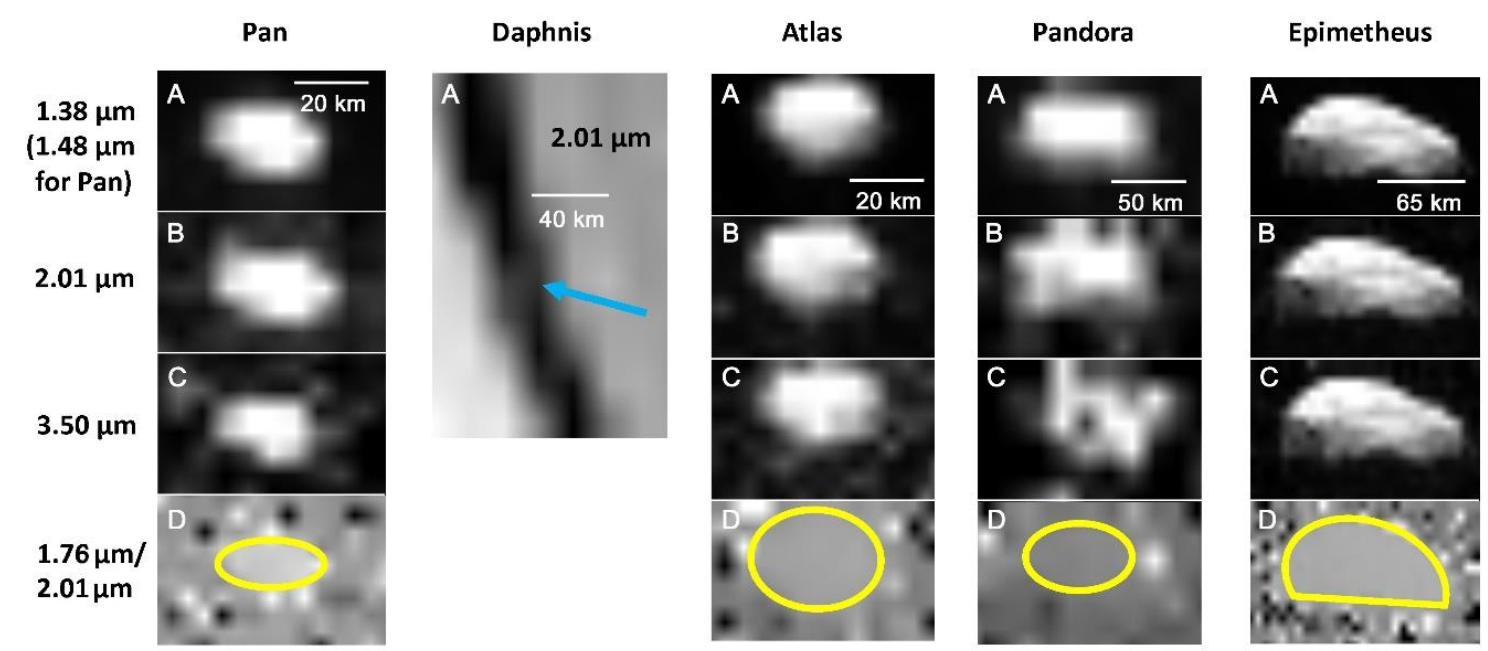

790

791

792

793

794

795

796

797

798

799

800

801

802

803

Fig. S1. Infrared images of the five ring moons studied during the Ring-grazing Orbits. A shows measurements at $1.38 \mu \mathrm{m}$ for Atlas, Pandora, and Epimetheus, $1.48 \mu \mathrm{m}$ for Pan, and 2.01 $\mu \mathrm{m}$ for Daphnis, which is shown by the blue arrow. B and C show $2.01 \mu \mathrm{m}$ and $3.50 \mu \mathrm{m}$, respectively. The bottom row is a ratio of the continuum at $1.76 \mu \mathrm{m}$ to the water ice absorption band at $2.01 \mu \mathrm{m}$, showing uniformity on all the moons' surfaces (the images for Daphnis were too noisy to construct this ratio). The yellow lines outline the approximate position of each moon.

\section{$\underline{\text { The Cassini Infrared Spectrometer Observations }}$}

The detections of both Atlas and Epimetheus were made using dedicated CIRS scans bracketed by ISS observations. Epimetheus was detected on 30 Jan 2017 during a scan that occurred between 19:54:20 to 20:05:50 UTC, at a distance that decreased from 80,179 to $67,237 \mathrm{~km}$. During this time the sub-spacecraft position changed from $345.0^{\circ} \mathrm{W} / 73.5^{\circ} \mathrm{N}$ to $346.5^{\circ} \mathrm{W} / 73.7^{\circ} \mathrm{N}$, the local time at the sub-spacecraft point increased from $271^{\circ}$ to $276^{\circ}$, and the solar phaseangle increased slightly from $68.0^{\circ}$ to $68.5^{\circ}$.

Atlas was detected a few months later, on 12 April 2017, during a scan that ran from 13:16:39 to 13:24:40 UT (Universal Time), at a distance that decreased from 33,572 km to 24,580 km. During 
that time the phase at the sub-spacecraft point decreased from $51.2^{\circ}$ to $47.2^{\circ}$, the sub-spacecraft position changed from $141.9^{\circ} \mathrm{W} / 60.1^{\circ} \mathrm{N}$ to $149.8^{\circ} \mathrm{W} / 52.1^{\circ} \mathrm{N}$, and the local time at the subspacecraft point decreased from $226^{\circ}$ to $221^{\circ}$.

In both detections CIRS used its focal plane 3 (FP3, which covers 8.9-17.5 $\mu \mathrm{m}$ ) to scan the target and background sky. The images have been rotated so they are also in RA/Dec coordinates. However, the scale of the CIRS data and the ISS images is notably different, as indicated by the $10 \mathrm{~km}$ scale bar given in Fig. 5 in the main text. Images of Atlas taken before and after the CIRS scan were ISS image N00279648 using CL1 and CL2 filters on Apr. 12, 2017 at 1:15 UT; ISS image N00279649 taken using CL1 and CL2 filters on Apr. 12, 2017 1:27 UT. Images of Epimetheus taken before and after the CIRS scan were ISS image N00275708 taken using CL1 and CL2 filters on Jan. 30, 2017 7:53 UT and ISS mage N00275709 taken using CL1 and UV3 filters on Jan. 30, 2017 8:07 UT.

\section{Supplementary Text}

\section{Overview of the Ring and Moon $\underline{\text { System of }} \underline{\text { Saturn }}$}

Saturn has 62 moons that group into several categories. Besides the five main inner moons (Mimas, Enceladus, Tethys, Dione, and Rhea), Hyperion, Titan, and Iapetus, the outer irregular moons, which include Phoebe, the planet has a family of ring moons that orbit in gaps within Saturn's rings (Pan in the Encke gap and Daphnis in the Keeler gap) or skirt the outer edge of the A-ring (Atlas) and the F-ring (Prometheus and Pandora). The coorbital moons Janus and Epimetheus, which exchange an orbit outside the A-ring approximately every four years, are often classified as ring moons as well. Fig. S2 illustrates the position of the ring moons within Saturn's system. 


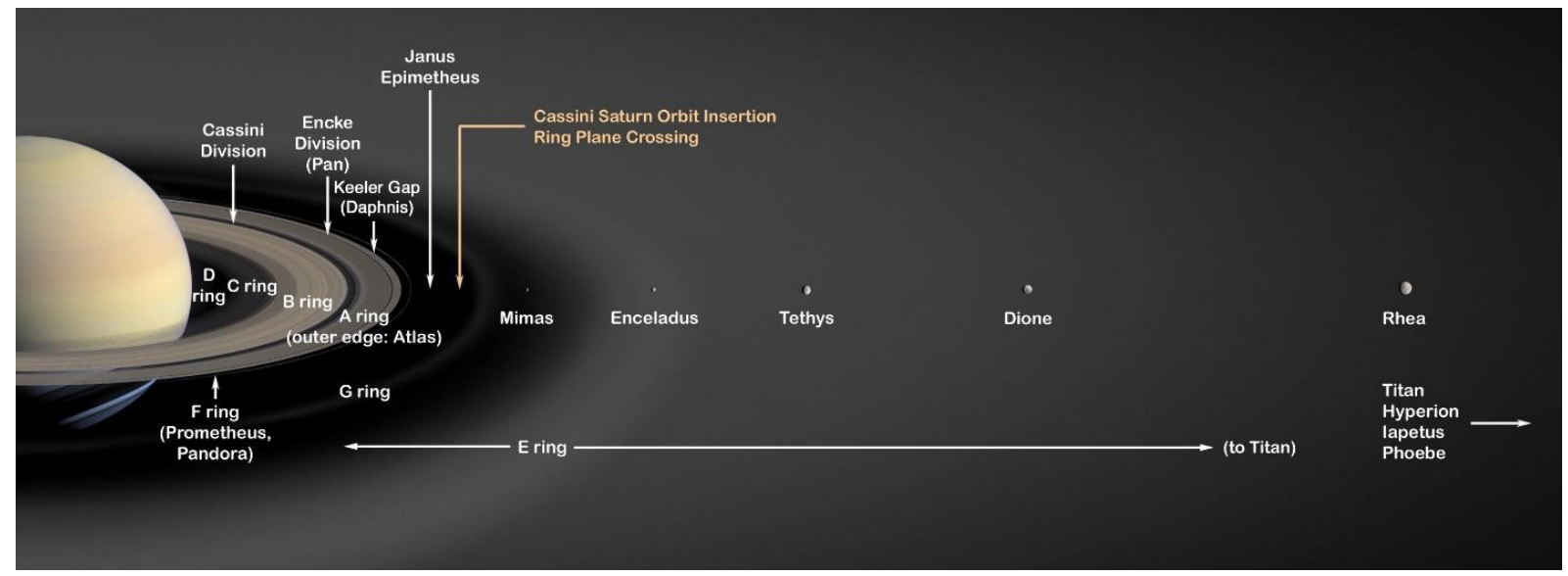

Figure S2. A diagram showing the location of the main ring system of Saturn, the main inner moons, and the ring moons Pan, Daphnis, Atlas, Pandora, and Prometheus. The coorbital moons Janus and Epimetheus are often regarded as ring moons as well. Based on NASA PIA03550

\section{1 (Public domain.)}

Numerical simulations and the lifetimes of the dust particles

We performed numerical simulations of dust particles in the Janus-Epimetheus ring to estimate their lifetimes. In Fig. S3, the solid line shows the fraction of remaining particles (those which did not yet collide with Janus, Epimetheus, Saturn or its dense rings). To obtain the mean lifetime $\tau$ of the dust particles, we fit an exponential function $f(t)=\exp (-t / \tau)$ to the simulation results,

837 shown as dashed line, yielding $\tau=60$ years.

We assume the dust particles to be spheres with a radius of $s_{d}=1.6 \mu \mathrm{m}$, which is consistent with the size of particles measured to comprise the Janus-Epimetheus ring by the HRD detector of Cassini's CDA (see main text). In the simulations, we consider the gravity of Saturn (including its oblateness up to 6th order), the gravity of Janus and Epimetheus, as well as solar radiation pressure 

summarizes the parameters used in the simulations.

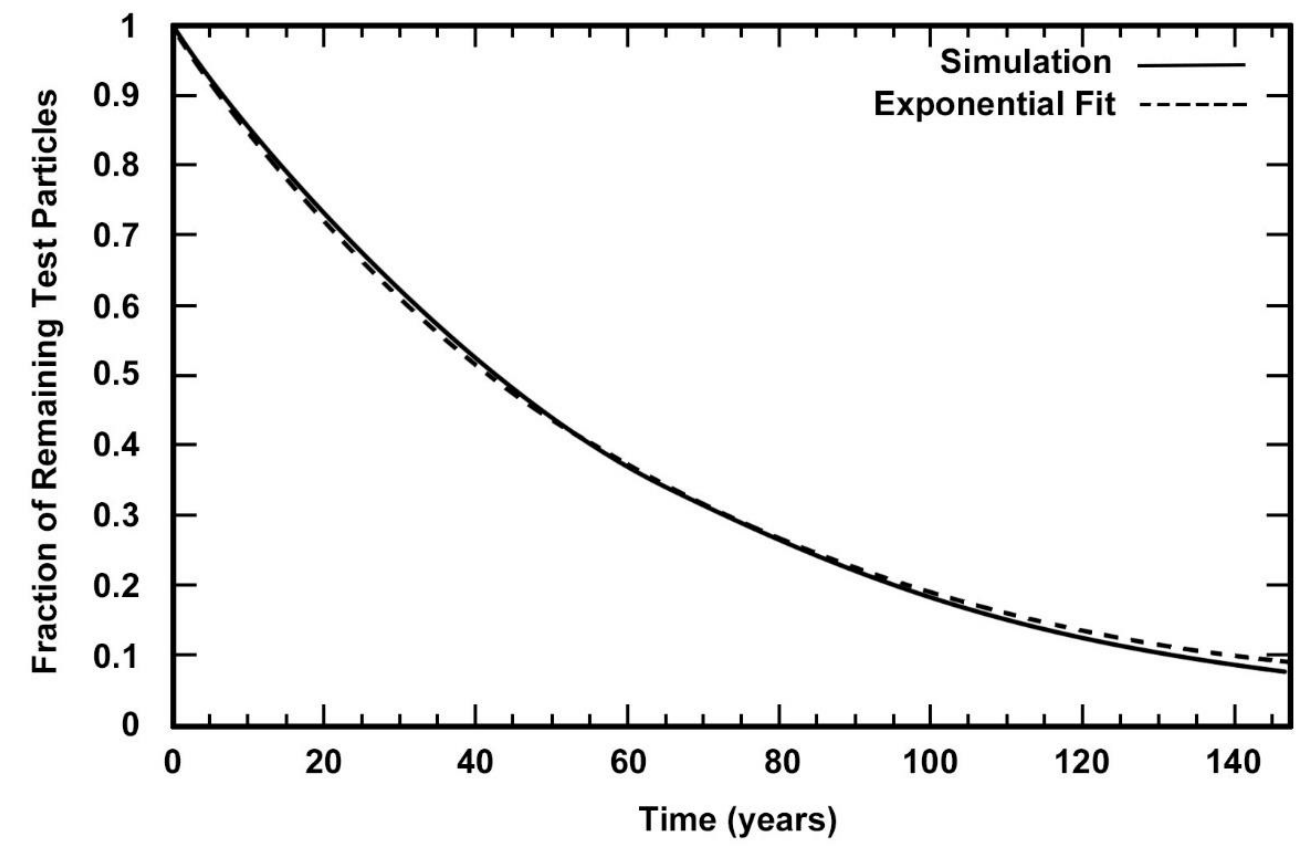

Fig S3. Particle Lifetimes. The solid line shows the evolution of the fraction of remaining particles

847 (which did not collide with Janus, Epimetheus, Saturn or its dense rings), whereas the dashed line 848 denotes an exponential fit to this evolution leading to a mean lifetime of $\tau=60$ years.

We integrated the equations of motion of 40,000 particles for about 150 years. For simplicity, the initial eccentricities and inclinations of the dust particles were chosen to be Rayleigh distributed with mean values of $\langle e\rangle=0.0068$ and $\langle i\rangle=0.17 \mathrm{deg}^{1}$, resembling a ring width of about $2000 \mathrm{~km}$ and a ring scale height of $350 \mathrm{~km}$. The initial ephemeris data of the Sun, Janus, and Epimetheus were obtained from data provided by the NAIF SPICE toolkit $(65,66)$ using the kernel files de430.bsp, sat375.bsp, sat378.bsp, and cpck23Aug2007.tpc.

\footnotetext{
${ }^{1}$ The initial argument of pericenters, longitude of the ascending nodes, and time of pericenter passages were uniformly distributed.
} 
857 The lifetime of the particles in the Janus and Epimetheus ring is also restricted by the surrounding 858 plasma, neglected in our simulations. The permanent bombardment of the dust particles by Saturn's 859 plasma particles leads to a sputtering of their surface, which reduces the size of the particles. The 860 typical plasma sputtering rate in the E-ring is about $1 \mu \mathrm{m}$ in 50 years (67). However, the plasma 861 density is decreasing by two orders of magnitude towards Saturn (68) which increases the 862 sputtering lifetime of a $1.6 \mu \mathrm{m}$ sized particle to $\tau_{\text {sputt }}=8000$ years.

864 Collisions with the plasma particles further accelerate the dust particles causing an outward drift 865 (plasma drag). While drift rates of $1000 \mathrm{~km} / \mathrm{yr}$ are typical in the E-ring for $1.6 \mu \mathrm{m}$ sized grains 866 (69), the drift rate in the Janus-Epimetheus region is only $10 \mathrm{~km} / \mathrm{yr}$ due to the lower plasma 867 densities (68). Therefore, a dust particle is estimated to leave the Janus-Epimetheus ring after about 868210 years, assuming a half width at half maximum of $2100 \mathrm{~km}$.

In summary, the collisions with the moons are the dominant sink for the ring particles leading to a

871 typical lifetime of about 60 years, which provides a fair explanation of the impact-generated ring 872 embracing the orbits of Janus and Epimetheus.

\section{Impact-ejection model}

874 It is assumed that the dust in the Janus-Epimetheus ring is generated by the process of impact875 ejection - the ejection of secondary dust particles by impacts of fast micro-meteoroids onto 876 atmosphereless planetary satellites. 
In order to estimate the dust densities in the ring, we apply the impact-ejection model (41). In this model, the total mass ejected from the target surface per unit time is given by

$$
M^{+}=F_{\text {imp }} Y S,
$$

where $F_{\mathrm{imp}}$ is the impactor mass flux (density) at the target and $S$ is the target's cross sectional area (41). $Y$ is the yield defined as the ratio of the total mass ejected by an impactor to its own mass. It strongly depends on the impact speed $v_{\mathrm{imp}}$ as well as the impactor mass $m_{\mathrm{imp}}$ and the composition of the target surface. We use an empirical relation for the yield (74), which reads (in SI units)

$$
Y=2.85 \times 10^{-8} \rho_{\text {ice }} m_{\mathrm{imp}}^{0.23} v_{\mathrm{imp}}^{2.46},
$$

where $\rho_{\text {ice }} \approx 930 \mathrm{~kg} / \mathrm{m}^{3}$ is the mass density of ice at a temperature of $100 \mathrm{~K}$.

The flux, sizes and speeds of the impactors have been obtained from in situ measurements of the Cassini CDA (43). At the Hill radius of Saturn, the impactor flux is $3.6 \times 10^{-16} \mathrm{~kg} \mathrm{~m}^{-2} \mathrm{~s}^{-1} \leq$ $F_{\text {imp }}^{\infty} \leq 4.2 \times 10^{-15} \mathrm{~kg} \mathrm{~m}^{-2} \mathrm{~s}^{-1}$, and the distributions of the impactor sizes and speeds can be fit by a log-normal distribution, respectively

$$
f(x)=\frac{1}{\sqrt{2 \pi} \sigma x} \exp \left(-\frac{(\log x-\mu)^{2}}{2 \sigma^{2}}\right),
$$

where the maximum and the mean are at $2.8 \mu \mathrm{m}$ and $5.8 \mu \mathrm{m}$ for the size distribution, and at $4.5 \mathrm{~km} / \mathrm{s}$ and $11 \mathrm{~km} / \mathrm{s}$ for the speed distribution. Because the impactor properties are widely distributed, all quantities (focused impactor flux, yield, total ejected mass) that depend on them are averaged over the impactor sizes and/or speeds, e.g.

$$
\bar{M}^{+}=\left\langle F_{\mathrm{imp}} Y S\right\rangle,
$$


where

$$
\langle\ldots\rangle=\int \ldots f(x) d x
$$

The impactor flux and speeds are amplified due to gravitational focusing by the planet $(42,75)$. At the planetocentric distance of Janus and Epimetheus $\left(2.5 \mathrm{R}_{\mathrm{S}}\right)$, the mean focusing factors are $\sim 4$ for the impact speeds and $\sim 23$ for the impactor flux, and the mean yield is $Y \sim 3800$. For the lower limit of the impactor flux, this corresponds to a mass production rate of $0.57 \mathrm{~kg} / \mathrm{s}$ for Janus and $0.24 \mathrm{~kg} / \mathrm{s}$ for Epimetheus.

The cumulative size distribution of the debris is assumed to be a power law with exponent $-\alpha$, so that the number of particles with radii larger than $s_{d}$ ejected from the target surface per unit time is given by

$$
N^{+}(>s)=\frac{3-\alpha}{\alpha} \frac{M^{+}}{m_{\max }}\left(\frac{s_{\mathrm{d}, \max }}{s_{d}}\right)^{\alpha}
$$

where $m_{\max }\left(s_{\mathrm{d}, \max }\right)$ is the maximal ejecta mass (size). The index $\alpha$ depends on the target material and ranges from 1.5 for loose to 3 for solid targets (76). In situ measurements give for the index of the size distribution values of $\alpha \sim 2.4$ for the dust atmospheres around the Galilean moons (44), and a value of $\alpha \sim 2.7$ for the lunar dust atmosphere (77). The largest ejecta is typically similar in size to the largest impactor (78). For $\alpha=2.4$ and $m_{\max }=10^{-8} \mathrm{~kg}$ (an icy particle with $s_{\mathrm{d}, \max } \approx$ $140 \mu \mathrm{m}), 6.4 \times 10^{11}$ particles larger than $1.6 \mu \mathrm{m}$ from Janus and $2.7 \times 10^{11}$ from Epimetheus are ejected per second. 
Impact experiments and scaling laws (79) show that the differential speed distribution is

922 proportional to a power law with exponent $-\gamma-1$

$$
f(u)=\frac{\gamma}{u_{\min }^{-\gamma}-u_{\max }^{-\gamma}} u^{-\gamma-1} \Theta\left(u-u_{\min }\right) \Theta\left(u_{\max }-u\right)
$$

where $\Theta(x)$ denotes the unit step function, which is one for $x \geq 0$ and zero otherwise. The index $\gamma$ depends on properties of the target material and ranges from $\gamma=1$ for highly porous to $\gamma=2$ for nonporous materials (41).

The minimal ejection speed $u_{\min }$ is chosen so that the kinetic energy of the ejecta is a few (tens of) percent of the kinetic energy of the impactor $(48,49)$. Hard surfaces (e.g. ice) are generally less dissipative than soft surfaces (e.g. snow, regolith). In case the ejecta sizes and ejection speeds are uncorrelated, the relation between $Y, \gamma$, and $u_{\min }$ reads $(80)$

$$
\frac{K_{\mathrm{e}}}{K_{\mathrm{imp}}}=Y \frac{\gamma}{2-\gamma}\left(\frac{u_{\mathrm{min}}}{v_{\mathrm{imp}}}\right)^{2}\left[\left(\frac{u_{\mathrm{min}}}{u_{\mathrm{max}}}\right)^{\gamma-2}-1\right] \text { for } \gamma \neq 2
$$

and

$$
\frac{K_{\mathrm{e}}}{K_{\mathrm{imp}}}=2 Y\left(\frac{u_{\mathrm{min}}}{v_{\mathrm{imp}}}\right)^{2} \ln \left(\frac{u_{\mathrm{max}}}{u_{\min }}\right) \text { for } \gamma=2,
$$

where the subscripts "imp" and " $e$ " refer to impactor and ejecta related variables, respectively.

The maximal ejection speed is larger than the escape velocity of the largest satellites in the Solar System $\left(u_{\max }>3 \mathrm{~km} / \mathrm{s}\right)$. For example, impact-ejecta escape the gravity of the Galilean moons and form a dust ring between their orbits (81). Integrating Equation (S7) for speeds larger than the escape velocity, $u>v_{\text {esc }}$, gives the fraction of escaping ejecta. 

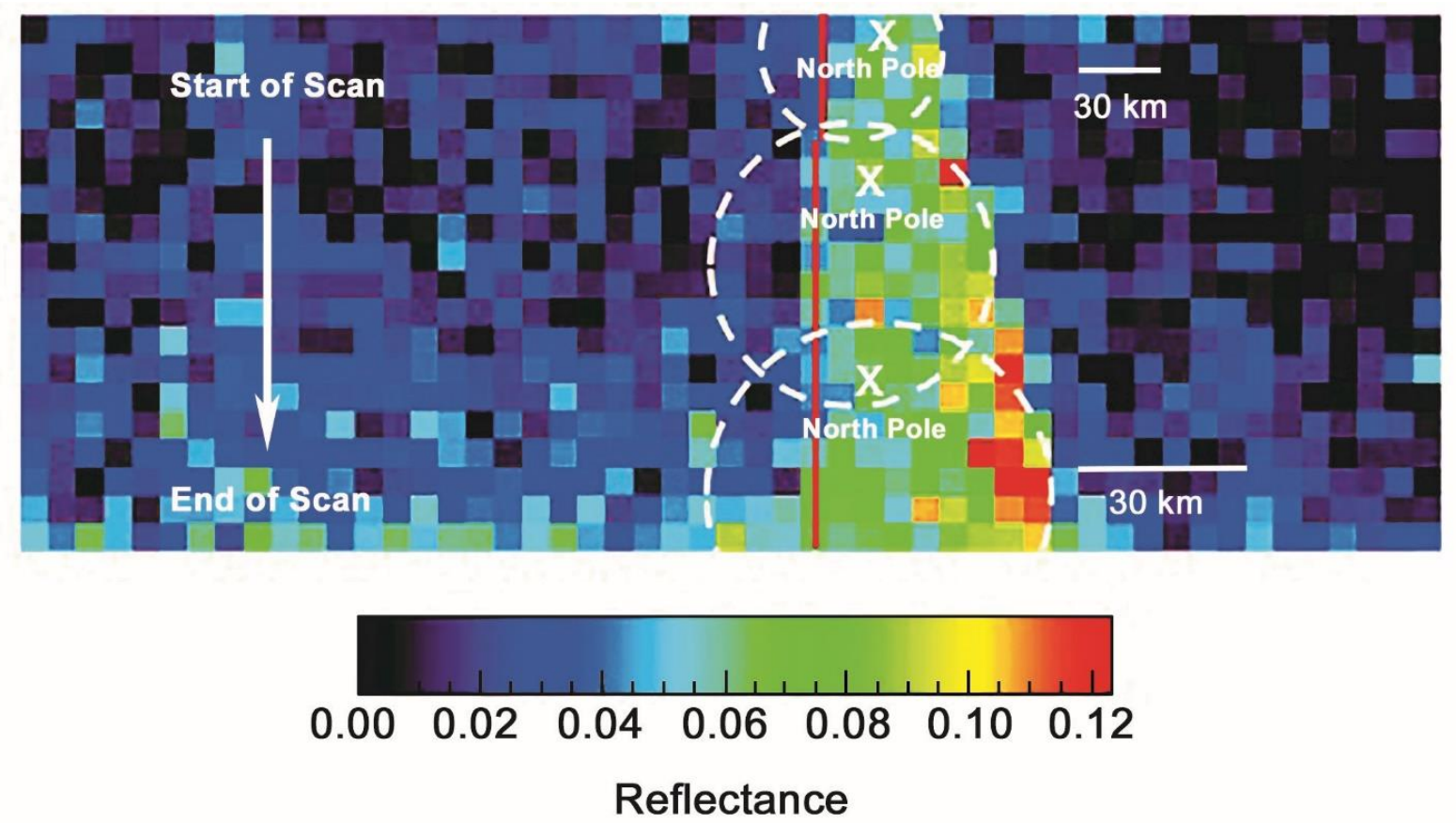

944

Fig. S4. Cassini UVIS scan of Epimetheus during the Feb 21, 2017 encounter. The altitude at the start of the scan was $\sim 18,773 \mathrm{~km}$ and at the end was $\sim 10,112 \mathrm{~km}$. White dashed lines indicate approximate location of the moon's limb and depict the changing relative size of Epimetheus throughout the scan. The red line indicates the approximate location of the terminator and " $\mathrm{x}$ " marks the approximate location of the north pole. Epimetheus (day and night sides) blocks the background interplanetary hydrogen. The wavelength is $0.170-0.190 \mu \mathrm{m}$ and each pixel is $1 \mathrm{mrad}$. 


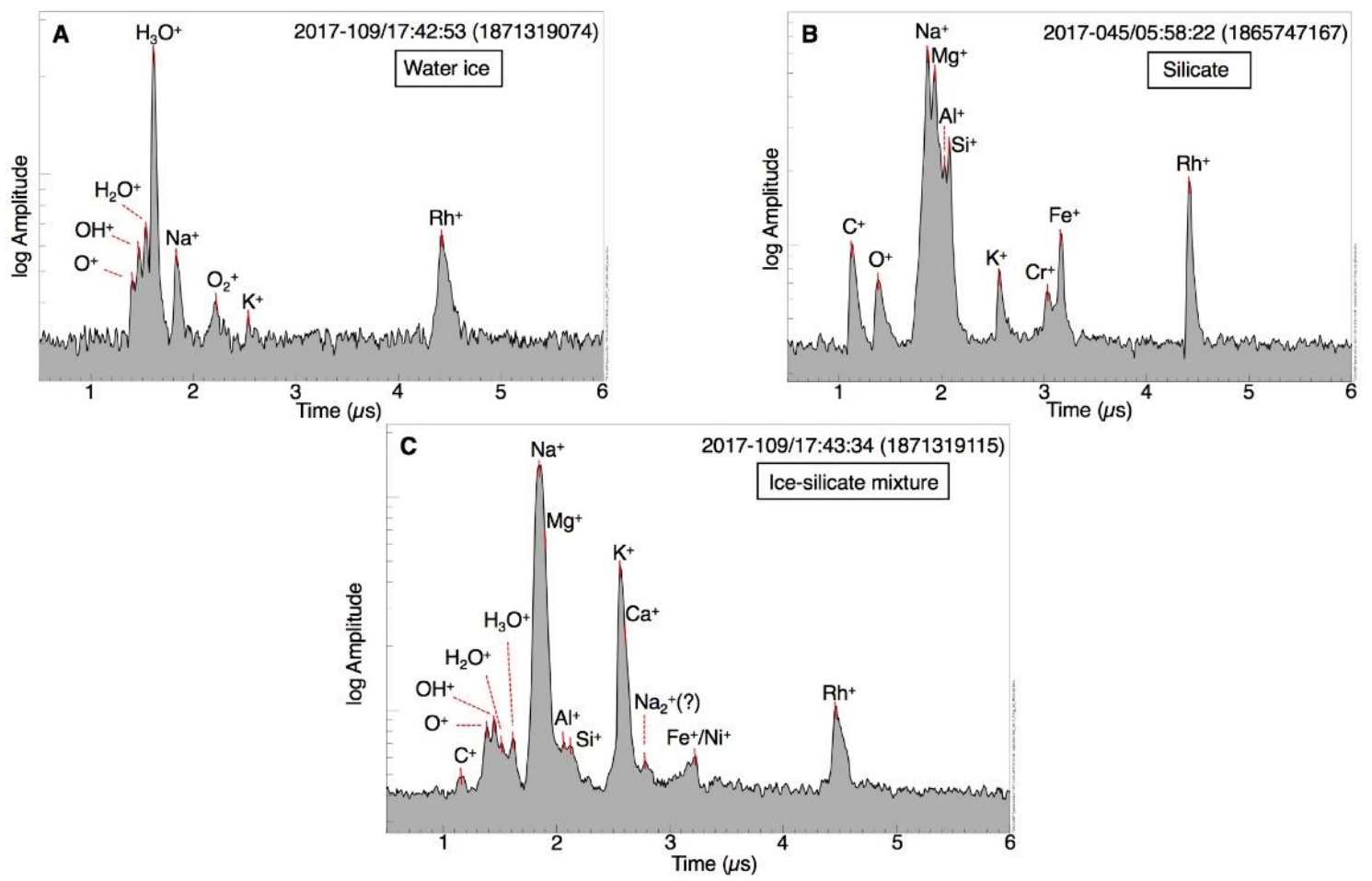

951

952

953

954

955

956

957

958

959

960

Fig S5. Example CDA mass spectra for the three populations of submicron grains detected

close to the plane of the $\mathbf{F}$ ring. The spectra recorded at impact velocities of approximately 20 $\mathrm{km} / \mathrm{s}$ over a few minutes around the ring plane on days 45 and 109 of the year 2017. Mass line $\mathrm{Rh}^{+}$ is from CDA's impact target material, rhodium. The $\mathrm{C}^{+}$mass line is due to ions from early target contamination, prior to Saturn orbit insertion (83), and those from $\mathrm{Na}+$ and $\mathrm{K}+$ are at least partially generated by ions from later target contamination from residues of myriads of salt-rich ice grain impacts during Enceladus plume crossings. However, contributions of $\mathrm{Na}$ and $\mathrm{K}$ cannot be excluded from the projectile material as well.

Panel A shows a spectrum from a nearly pure water-ice grain, characterized by mass lines from $\mathrm{H}_{3} \mathrm{O}^{+}, \mathrm{H}_{2} \mathrm{O}^{+}, \mathrm{OH}^{+}, \mathrm{O}^{+}$, and $\mathrm{O}_{2}{ }^{+}$. Panel $\mathrm{B}$ shows the spectrum of a silicate grain, characterized by mass lines from $\mathrm{Si}^{+}, \mathrm{O}^{+}, \mathrm{Mg}^{+}, \mathrm{Al}^{+}, \mathrm{Cr}^{+}$, and $\mathrm{Fe}^{+}$. The spectrum in panel $\mathrm{C}$ shows characteristic mass lines of both silicate and water ice: $\mathrm{Fe}^{+}, \mathrm{Ni}^{+} \mathrm{Mg}^{+}, \mathrm{Ca}^{+}, \mathrm{Al}^{+}, \mathrm{Si}^{+}$together with $\mathrm{H}_{3} \mathrm{O}^{+}, \mathrm{H}_{2} \mathrm{O}^{+}$, 
$\mathrm{OH}^{+}$and $\mathrm{O}^{+}$, and hence is identified as a mixed-phase grain. Here, $\mathrm{Mg}, \mathrm{Ca}$ and Ni cannot be clearly separated from the neighboring $\mathrm{Na}, \mathrm{K}$, and Fe peaks, respectively. A mass line labeled as $\mathrm{Na}_{2}^{+}$ could be due to a particularly high abundance of $\mathrm{Na}$ ions here.

Note that the abundances of the cations do not necessarily correspond to the elemental abundances in the dust grain, as ionization efficiencies for the observed species vary drastically (84). For example, cation-forming metals, such as $\mathrm{Na}$ and $\mathrm{K}$, form substantial cationic peaks at grain concentrations at which $\mathrm{Si}$ and $\mathrm{O}$ remain undetectable.

Table S1 Sizes and mean densities of Saturn's small moons

972

$\mathrm{A}, \mathrm{b}$, and $\mathrm{c}$ are the semimajor axes of the moons, and $\mathrm{R}_{\mathrm{m}}$ is the radius of a sphere of equivalent volume. Shape models of all the moons, except for ellipsoidal satellites Aegaeon, Methone, and Pallene, and the poorly resolved Polydeuces, and supporting information including image sources are available from the Planetary Data System's Small Body Node (https://sbn.psi.edu/pds/archive/sat.html). Ellipsoidal values are derived in $(6)$.

978

\begin{tabular}{|l|l|l|l|l|l|l|}
\hline Object & $\mathrm{a}, \mathrm{km}$ & $\mathrm{b}, \mathrm{km}$ & $\mathrm{c}, \mathrm{km}$ & $\mathrm{R}_{\mathrm{m}}, \mathrm{km}$ & $\begin{array}{l}\text { Density, } \\
\mathrm{kg} \mathrm{m}^{-3}\end{array}$ & $\begin{array}{l}\text { Surface } \\
\text { gravity range, } \\
\mathrm{cm} \mathrm{s}^{-2}\end{array}$ \\
\hline Pan & $17.3 \pm 0.2$ & $14.1 \pm 0.2$ & $10.5 \pm 0.7$ & $13.7 \pm 0.3$ & $400 \pm 32$ & $0.2-1.7$ \\
\hline Daphnis & $4.9 \pm 0.3$ & $4.2 \pm 0.8$ & $2.8 \pm 0.6$ & $3.9 \pm 0.5$ & $274 \pm 142$ & $0.0-0.4$ \\
\hline Atlas & $20.4 \pm 0.1$ & $17.7 \pm 0.2$ & $9.3 \pm 0.3$ & $14.9 \pm 0.2$ & $412 \pm 19$ & $0.0-1.7$ \\
\hline Pandora & $51.5 \pm 0.3$ & $39.5 \pm 0.3$ & $31.5 \pm 0.2$ & $40.0 \pm 0.3$ & $509 \pm 12$ & $2.0-5.9$ \\
\hline Epimetheus & $64.8 \pm 0.4$ & $58.1 \pm 0.8$ & $53.5 \pm 0.4$ & $58.6 \pm 0.5$ & $625 \pm 16$ & $6.6-10.9$ \\
\hline Janus & $101.8 \pm 0.9$ & $93.0 \pm 0.3$ & $74.5 \pm 0.3$ & $89.0 \pm 0.5$ & $642 \pm 10$ & $10.9-16.9$ \\
\hline Aegaeon & $0.7 \pm 0.0$ & $0.3 \pm 0.1$ & $0.2 \pm 0.0$ & $0.3 \pm 0.0$ & $539 \pm 140$ & $0.001-0.005$ \\
\hline Methone & $1.9 \pm 0.0$ & $1.3 \pm 0.0$ & $1.2 \pm 0.0$ & $1.4 \pm 0.0$ & $307 \pm 30$ & $0.1-0.1$ \\
\hline Pallene & $2.9 \pm 0.4$ & $2.1 \pm 0.3$ & $1.8 \pm 0.3$ & $2.2 \pm 0.3$ & $251 \pm 75$ & $0.1-0.2$ \\
\hline Telesto & $16.6 \pm 0.3$ & $11.7 \pm 0.3$ & $9.6 \pm 0.2$ & $12.3 \pm 0.3$ & & \\
\hline Calypso & $14.7 \pm 0.3$ & $9.3 \pm 0.9$ & $6.4 \pm 0.3$ & $9.5 \pm 0.4$ & & \\
\hline Polydeuces & $1.5 \pm 0.3$ & $1.3 \pm 0.4$ & $1.0 \pm 0.2$ & $1.3 \pm 0.3$ & & \\
\hline Helene & $22.6 \pm 0.2$ & $19.6 \pm 0.3$ & $13.3 \pm 0.2$ & $18.1 \pm 0.2$ & & \\
\hline
\end{tabular}


Table S2: Cassini ISS images used for color ratios.

981 These images and and corresponding observation geometry data were used to measure the 982 IR3/UV3 color ratios of Pan, Daphnis, Atlas, and Pandora. The rings were measured from the 983 same images as Pandora.

\begin{tabular}{|l|c|c|c|c|c|c|c|}
\hline Object & $\begin{array}{c}\text { ISS } \\
\text { Filter }\end{array}$ & $\begin{array}{c}\text { Image } \\
\text { Number }\end{array}$ & $\begin{array}{c}\text { Cassini } \\
\text { Range } \\
\text { (km) }\end{array}$ & $\begin{array}{c}\text { Pixel } \\
\text { Scale } \\
(\mathrm{m} / \text { pix })\end{array}$ & $\begin{array}{c}\text { Cassini } \\
\text { (latitude, } \\
\text { longitude) }\end{array}$ & $\begin{array}{c}\text { Sun } \\
\text { (latitude, } \\
\text { longitude) }\end{array}$ & $\begin{array}{c}\text { Phase } \\
\text { Angle }\end{array}$ \\
\hline Pan & UV3 & N1867604558 & 25639 & 150 & $44^{\circ} \mathrm{N}, 214^{\circ} \mathrm{W}$ & $27^{\circ} \mathrm{N}, 199^{\circ} \mathrm{W}$ & $21^{\circ}$ \\
\hline & IR3 & $\mathrm{N} 1867604614$ & 25048 & 147 & $42^{\circ} \mathrm{N}, 215^{\circ} \mathrm{W}$ & $27^{\circ} \mathrm{N}, 199^{\circ} \mathrm{W}$ & $20^{\circ}$ \\
\hline & & & & & & & \\
\hline Daphnis & $\mathrm{UV} 3$ & $\mathrm{~N} 1863267280$ & 27953 & 164 & $12^{\circ} \mathrm{N}, 116^{\circ} \mathrm{W}$ & $27^{\circ} \mathrm{N}, 191^{\circ} \mathrm{W}$ & $71^{\circ}$ \\
\hline & IR3 & $\mathrm{N} 1863267342$ & 26772 & 157 & $10^{\circ} \mathrm{N}, 116^{\circ} \mathrm{W}$ & $27^{\circ} \mathrm{N}, 191^{\circ} \mathrm{W}$ & $72^{\circ}$ \\
\hline & & & & & & & \\
\hline Atlas & UV3 & $\mathrm{N} 1870698966$ & 20060 & 118 & $44^{\circ} \mathrm{N}, 143^{\circ} \mathrm{W}$ & $27^{\circ} \mathrm{N}, 190^{\circ} \mathrm{W}$ & $41^{\circ}$ \\
\hline & IR3 & $\mathrm{N} 1870699087$ & 18013 & 106 & $40^{\circ} \mathrm{N}, 146^{\circ} \mathrm{W}$ & $27^{\circ} \mathrm{N}, 190^{\circ} \mathrm{W}$ & $39^{\circ}$ \\
\hline & & & & & & & \\
\hline Pandora & UV3 & $\mathrm{N} 1860790502$ & 42150 & 247 & $36^{\circ} \mathrm{N}, 98^{\circ} \mathrm{W}$ & $27^{\circ} \mathrm{N}, 185^{\circ} \mathrm{W}$ & $72^{\circ}$ \\
\hline & IR3 & $\mathrm{N} 1860792229$ & 22128 & 130 & $21^{\circ} \mathrm{S}, 106^{\circ} \mathrm{W}$ & $27^{\circ} \mathrm{N}, 196^{\circ} \mathrm{W}$ & $100^{\circ}$ \\
\hline
\end{tabular}

984

985

986

987

988

989

990

\section{Table S3. Parameters used in the numerical simulations}

The parameters are: solar radiation pressure efficiency factor $Q_{\mathrm{pr}}$, solar constant $Q_{\mathrm{s}}$, electrostatic grain potential $\phi_{\text {grain }}$, dipole term of Saturn's magnetic field $g_{10}$, and the gravitational harmonic coefficient $J_{2}, J_{4}$, and $J_{6}$.

\begin{tabular}{ccc}
\hline Parameter & Value & Reference \\
\hline Radiation Pressure: & 0.49 & $(70)$ \\
$Q_{\mathrm{pr}}$ & $1.36 \times 10^{3} \mathrm{Wm}^{-2}$ & \\
$Q_{\mathrm{s}}$ &
\end{tabular}

Lorentz Force:

$\begin{array}{ccc}\phi_{\text {grain }} & -1.6 \mathrm{~V} & (71) \\ g_{10} & 2.1162 \times 10^{-5} \mathrm{~T}\end{array}$

Saturn's Oblateness:

$\begin{array}{cc}J_{2} & 1.629071 \times 10^{-2} \\ J_{4} & -9.3583 \times 10^{-4} \\ J_{6} & 8.614 \times 10^{-5}\end{array}$

991

992

993 
995 Table S4. Parameters and results used for the impact-ejection model.

\begin{tabular}{lcl}
\hline Parameter & Value & Reference/Comment \\
\hline$F_{\text {imp }}^{\infty}$ & $3.6 \times 10^{-16} \mathrm{~kg} \mathrm{~m}^{-2} \mathrm{~s}^{-1}$ & $(43)$ \\
$\bar{F}_{\text {imp }}$ & $8.2 \times 10^{-15} \mathrm{~kg} \mathrm{~m}^{-2} \mathrm{~s}^{-1}$ & \\
$\bar{Y}$ & 3800 & \\
$\bar{M}^{+}$ & $0.8 \mathrm{~kg} \mathrm{~s}^{-1}$ & $70 \%$ Janus and $30 \%$ Epimetheus \\
$\alpha$ & 2.4 & $(44)$ \\
$m_{\max }$ & $1.0 \times 10^{-8} \mathrm{~kg}$ & $(44)$ \\
$N^{+}(>s)$ & $9.1 \times 10^{11} \mathrm{~s}^{-1}$ & $70 \%$ Janus and $30 \%$ Epimetheus \\
$K_{\mathrm{e}} / K_{\mathrm{imp}}$ & 0.05 & $\begin{array}{l}\text { Exact agreement of the estimated number } \\
\end{array}$ \\
& & of particles in the Janus-Epimetheus ring \\
& & with the observed value would require a \\
& & very small kinetic energy ratio of ejecta to \\
& & impactor of about $1 \%$. \\
$\gamma$ & 1.0 & $(41)$ \\
$N_{\text {esc }}^{+}(>s)$ & $5.2 \times 10^{10} \mathrm{~s}^{-1}$ & $60 \%$ Janus and $40 \%$ Epimetheus \\
\hline
\end{tabular}

996

997

998 